\title{
Management of Impacted Third Molars
}

\author{
George Varghese
}

\subsection{Introduction}

Even as the scope of practicing oral and maxillofacial surgery grows and continues to evolve, the mainstay of practice remains dentoalveolar surgery. In this area, the surgical removal of impacted teeth is one of the commonest procedures that is performed. Among the teeth that are commonly impacted, the mandibular molars rank first, followed by the maxillary third molars and the maxillary canines. Less commonly, impaction of other teeth such as the mandibular canines, maxillary and mandibular premolars, and the second molars are also seen.

\subsubsection{Terminology}

The term impaction comes from the term "impactus," which is of Latin origin. Its general usage refers to the failure of an organ or structure in achieving its normal position because of an abnormal mechanical condition.

Archer [1] defined impacted tooth as a tooth that is partially or completely unerupted and is positioned against another tooth or bone or soft tissue so that its further eruption is unlikely.

Lytle [2] proposed a definition that is intimately related to that of Archer. An impacted tooth is a tooth that has failed to erupt into its normal functional position beyond the time usually expected for such appearance. Eruption may have been prevented by adjacent hard or soft tissue including tooth, bone, or dense soft tissue.

Electronic Supplementary Material The online version of this chapter (https://doi.org/10.1007/978-981-15-1346-6_14) contains supplementary material, which is available to authorized users.
Andreasen et al. [3] defined impaction as a cessation of the eruption of a tooth caused by a clinically or radiographically detectable physical impediment in the eruption path or by an ectopic position of the tooth.

- An unerupted tooth is the one lying within the jaws, entirely covered by soft tissue, and partially or completely covered by bone. This tooth is undergoing the eruption process and will probably erupt into occlusion based on clinical and radiographic findings.

- A partially erupted tooth is one that has failed to erupt fully into a normal position. The term implies that the tooth is partly visible or in communication with the oral cavity.

- An impacted tooth is a tooth which is prevented from completely erupting into a normal functional position. The reason may be due to lack of space, obstruction by another tooth, or an abnormal eruption path.

\subsubsection{Incidence of Impaction}

Archer observed that the following types of teeth, in order of frequency, are most likely to be impacted: maxillary third molars, mandibular third molars, maxillary cuspids, mandibular bicuspids, mandibular cuspids, maxillary bicuspids, maxillary central incisors, maxillary lateral incisors.

G. Varghese $(\bowtie)$

Principal, Professor and Head, Department of Oral and

Maxillofacial Surgery, Pushpagiri College of Dental Sciences,

Tiruvalla, Kerala, India 


\subsection{Management Techniques for the Impacted Tooth}

Although the standard management strategy is usually considered to be surgical removal of the impacted tooth, the following methods listed below also should be considered depending upon the case:

1. Conservative method-Leaving the tooth alone with regular follow-up clinically and radiographically. For instance, a deeply asymptomatic third molar may be left as such especially in an older age group patient.

2. Operculectomy-This procedure can be considered in a mandibular third molar that has partially erupted, and has sufficient space to come into occlusion, but is prevented from doing so by thick overlying mucoperiosteum. If the tooth still fails to erupt fully, it has to be considered for removal.

3. Autogenous transplantation-Occasionally, the third molars can be considered for autogenous transplantation, usually to a first molar socket site. Because of the low success rate with such procedures, it is not widely used except in special circumstances.

4. Orthodontically guided eruption-This is usually suited to impacted maxillary and mandibular canine teeth. Orthodontic guidance enables the tooth to reach a functional position within the arch. This technique can also be applied to impacted premolars and, in some instances, even impacted mandibular molars.

5. Procedures that activate eruption-When indicated, these are usually applied to developing teeth.

\subsubsection{Controversies on Prophylactic Removal of Third Molars}

The benefits of prophylactic surgical removal of impacted third molars that are disease-free is quite controversial [4-6]. There are opinions that retaining the teeth may be more costeffective than prophylactic removal, at least in the short to medium term. Nevertheless, there may still be clinical situations that demand prophylactic surgery. Each clinical scenario needs an individualized evaluation and the consequences of all management techniques must be discussed with the patient. Thomas Dodson in [4] brought out a classification based upon the presence/absence of symptoms and the presence/absence of disease. He proposed to use this method to decide on the removal vs retention of third molars.

\subsection{Etiology of Impaction}

Generally, the third molars or the wisdom teeth are the last teeth to erupt and they erupt between 18 and 25 years of age. Since they erupt at about the time when the youth goes off into the world to become "wise," the name "wisdom teeth" was used.

A number of theories have been put forth to explain the phenomenon of impaction. The following are the most commonly accepted ones:-

1. Discrepancy between the tooth size and the arch length.

2. Differential growth pattern of the mesial and distal roots.

3. Delayed maturation of the third molar-dental development of the tooth lags behind the skeletal growth and maturation.

4. Incidence of extraction of permanent molars is decreased in the mixed dentition period, providing less room for eruption of third molars. This is very pertinent in the present day due to better awareness of the population and dental treatments are started early in childhood.

5. Inadequate development of jawbones due to consumption of more refined food which causes reduced functional stimulation for the growth of jaw bone.

6. Evolution theory.

Berger [7] listed the following local causes for impaction of teeth:

1. Irregularity in the position and pressure of an adjacent tooth.

2. The increased density of the overlying or surrounding bone.

3. Continued chronic inflammation with subsequent increase in density of the overlying mucous membrane.

4. Lack of space due to underdeveloped jaws.

5. Prolonged retention of the primary tooth.

6. Early loss of primary tooth.

7. Acquired diseases such as necrosis due to infection or abscess.

Impaction may also be found with no local predisposing conditions cited above.

According to Berger, the following are the systemic causes of impaction:

(a) Prenatal causes-Hereditary and miscegenation.

(b) Postnatal causes-Rickets, anemia, congenital syphilis, tuberculosis, endocrine dysfunction, and malnutrition.

(c) Rare conditions-Cleidocranial dysostosis, oxycephaly, progeria, achondroplasia, and cleft palate.

\subsection{Indications for Removal}

Despite the fact not all unerupted/impacted teeth cause problems, all have that potential. Based on extensive clinical studies, indications for removal have been identified.

1. Pericoronitis and Pericoronal abscess (Fig. 14.1a, b, c) - This is the most common cause for extraction of mandibular third molars (25-30\%). Pericoronitis is frequently found to be associated with distoangular and 

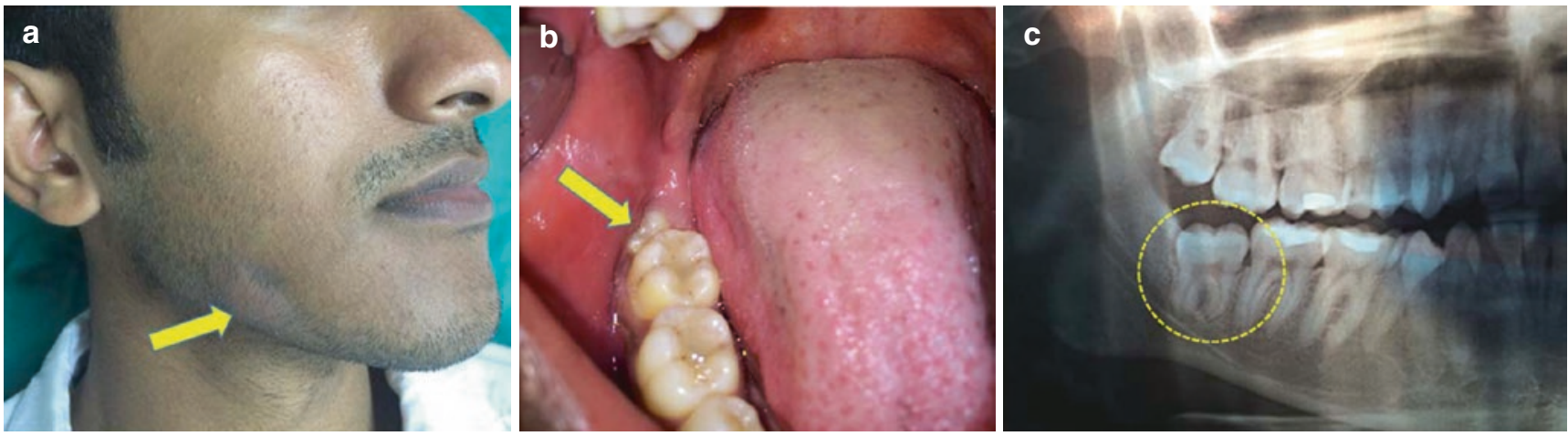

CAssociation of Oral and Maxillofacial Surgeons of India

Fig. 14.1 (a, b, c) Impacted right mandibular third molar with pericoronitis causing extraoral abscess (a) Extraoral abscess (Yellow arrow), (b) Impacted 48 with pericoronitis (yellow arrow), (c) OPG showing impacted 48 (Yellow circle)

vertical impaction. If treated inadequately, the infection may extent posteriorly resulting in submasseteric abscess.

2. Dental Caries-Incidence of caries of the second molar or third molar is about $15 \%$. The reason for this high incidence is attributed to difficulty to perform oral hygiene measures in the third molar area (Fig. 14.2).

3. Periodontal diseases-Repeated food impaction and collection of food debris between the impacted third molar and the erupted second molar can lead to periodontal disease and subsequent bone loss. This weakens the bone support for the second molar and can cause pulpo-periodontal disease in the second molar (Fig. 14.3).

\section{Orthodontic reasons}

(a) Crowding of incisors: Third molars has the potential to generate force in an anterior direction, which in turn can cause mandibular incisor crowding. Hence, removal of third molars has been recommended during or after orthodontic treatment. The hypothesis that the mesial pressure from the third molars is transferred through the contact points resulting in the narrow contacts of the lower incisors is slipping. Contemporary studies have questioned this hypothesis. However, it is still believed by certain clinicians, and third molars may be removed for these reasons.

(b) To facilitate orthodontic treatment-Since the recent trends in orthodontics prefer non-extraction modalities of treatment, distalization of molars has become ever more popular, particularly with regard to Class II malocclusions. In such cases, in order to expedite the distal movement of maxillary molars, the impacted or erupted maxillary third molar tooth may be extracted.

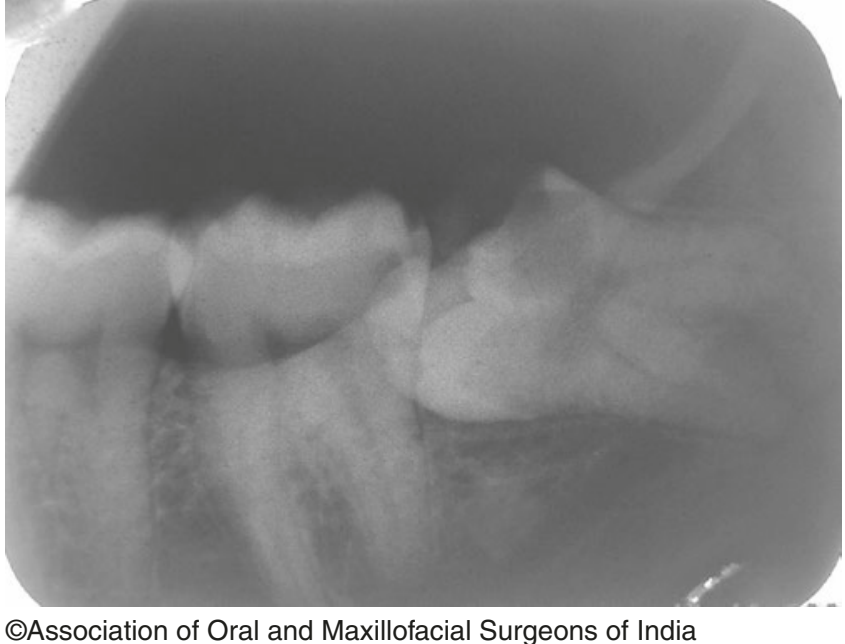

Fig. 14.2 IOPA X-ray of Horizontally impacted tooth 38 with dental caries

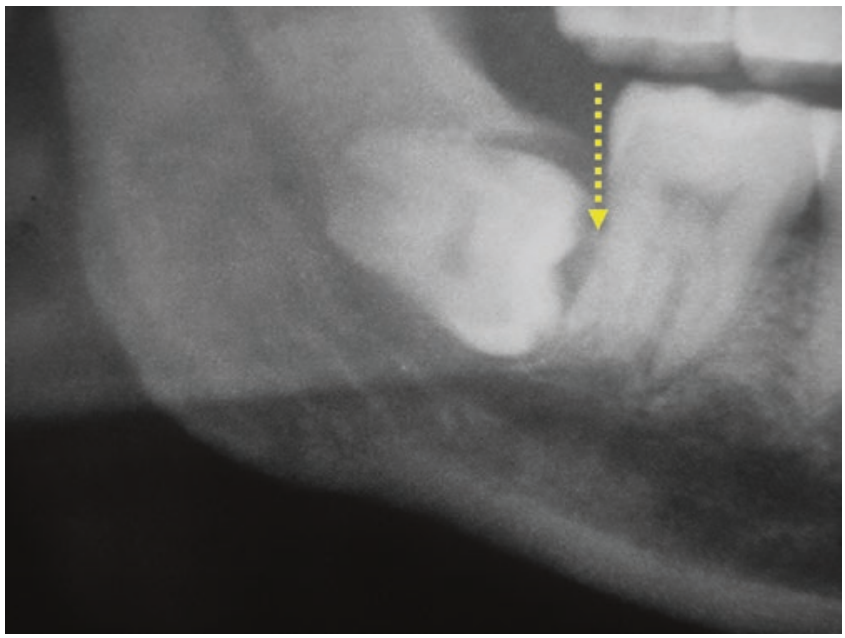

CAssociation of Oral and Maxillofacial Surgeons of India

Fig. 14.3 Horizontal impaction of 48 causing bone loss (yellow arrow) distal to 47 


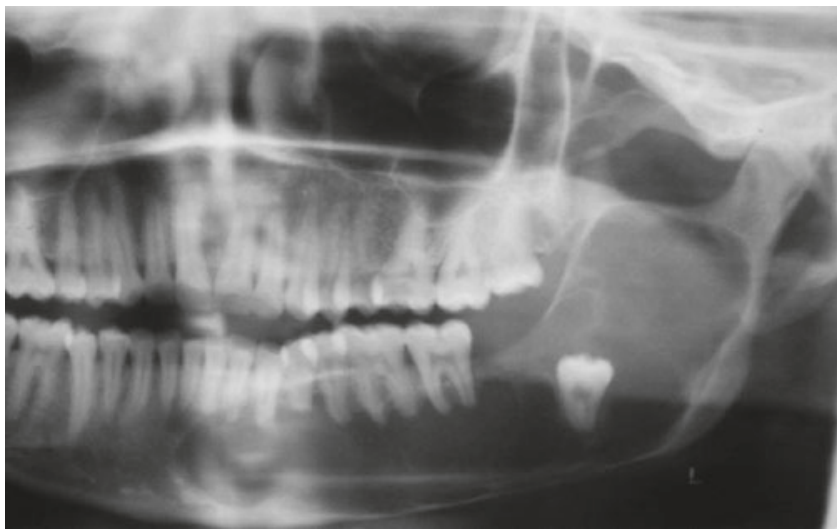

CAssociation of Oral and Maxillofacial Surgeons of India

Fig. 14.4 OPG showing impacted 38 associated with dentigerous cyst of mandible involving the left ramus, angle, and body

5. To facilitate orthognathic surgery-Removal of third molars should be considered in the presurgical preparation for orthognathic surgery. Making bone cuts in Bilateral sagittal split osteotomies (BSSO) is easier after third molars are removed. To ensure that adequate bone exists in this region, these must be extracted at least one year before the planned osteotomy.

6. Odontogenic cysts and tumors-Cysts and tumors may develop from the retained follicle around the impacted tooth (Fig. 14.4). To prevent this, extraction of asymptomatic third molars is recommended.

7. Unexplained pain-Sometimes, unexplained pain may be alleviated simply by removing impacted teeth, although the mechanism is still unclear. However, the pros and cons must be discussed with the patient.

8. Resorption of the adjacent tooth root-Pressure from the impacted tooth can cause the root of the second molar to resorb. When this is identified, the third molar must be removed as early as possible to avoid further damage.

9. For placement of dental prosthesis-The removal of impacted teeth under dental prosthesis must be assessed carefully, with the evaluation of risk versus benefits. Removal may be done for teeth that are superficial. However, sometimes the impacted tooth may lie deep within the mandible and in such cases, the tooth is better off left in situ.

10. Prevention of jaw fracture-For those engaged in contact games, it may be better to prophylactically remove the impacted third molars, as this area may be prone to fracture due to lowered bone resistance (Fig. 14.5).

11. Infection of deep fascial spaces-When pericoronitis is associated with impacted tooth, infection can track into deep fascial spaces.

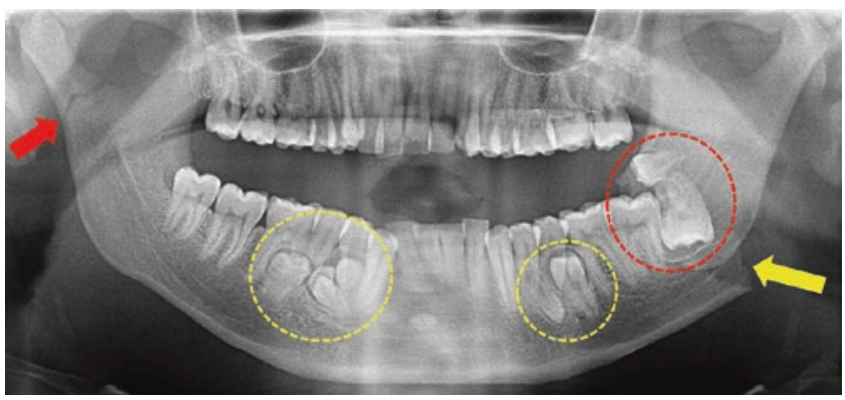

CAssociation of Oral and Maxillofacial Surgeons of India

Fig. 14.5 OPG showing impacted left mandibular third molar in inverted position associated with supernumerary (red circle) with fracture of left angle mandible (yellow arrow). Note multiple impacted supernumeraries (yellow circles) and fracture of right condyle (red arrow)

12. To remove a potential infection source (e.g. prior to administration of radiotherapy)_-Teeth which are at risk of infection like partially erupted third molar tooth may lead to local complications like osteoradionecrosis or systemic complications like endocarditis. Removal must be considered for these cases as well as other procedures such as chemotherapy, organ transplantation, or insertion of alloplastic implants.

13. Removal for autogenous transplantation-Even though this was a very popular procedure in the past, it fell into disrepute due to unpredictable results. However, it is worth considering when indicated for first molar replacements.

\subsubsection{Relative Contraindications for Removal of Impacted Tooth}

1. Compromised systemic status-It may not be advisable to undertake surgical removal of impacted third molars in patients with uncontrolled or poorly controlled systemic disease, as they can develop complications during or after the procedure. Hence, a proper history, physical examination, and, if needed, appropriate laboratory investigations must be performed.

2. Advanced age-Bone sclerosis increases with advancing age. This leads to poor healing, a larger defect size, and increased difficulty of the procedure. Risk of mandibular fracture is also high in these cases.

3. Damage to any adjacent structures-If the inferior alveolar canal is in close contact with the impacted tooth, inadvertent damage can result in paresthesia.

4. Questionable status of the second molar-If the second molar is badly decayed and unrestorable, removing it may allow the third molar to come into a 


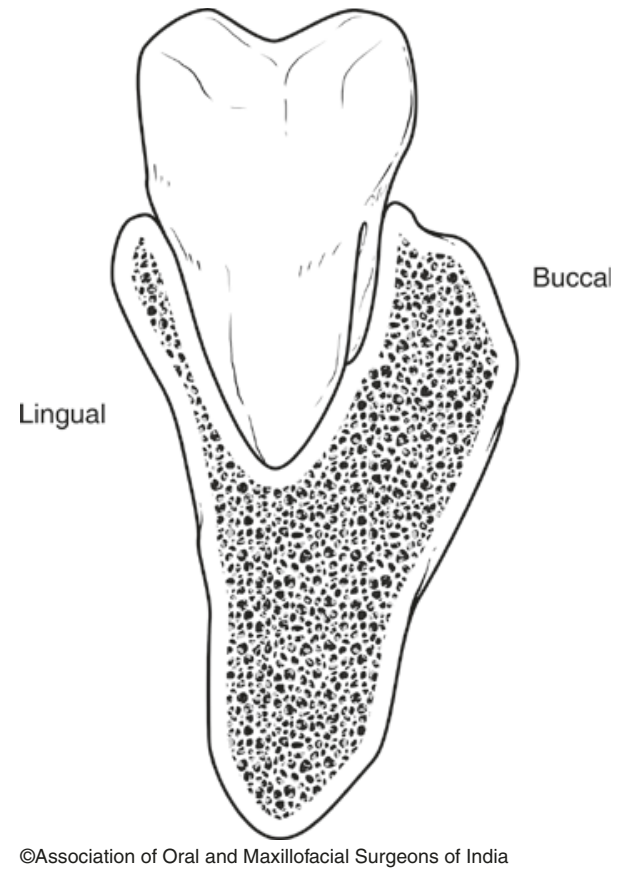

Fig. 14.6 Coronal section of mandible in the region of the third molar showing a thick buccal alveolar bone and a thin lingual plate

functional position. The third molar may also serve as a bridge abutment. Such cases require multidisciplinary evaluation with the prosthodontist and endodontist.

5. Deeply impacted third molars which do not appear to be associated with local or systemic pathology must not be removed.

\subsection{Surgical Anatomy}

The mandible comprises of a body which is horseshoe shaped and has the ramus on either side, which are flat and broad rami. Each ramus has two processes at the superior end - the coronoid process, which is more anterior, and condylar process, which is continuous with the posterior border.

The mandibular third molar tooth is usually present at the distal end of the mandibular body, which adjoins a thin ramus. The body-ramus junction is a weak area that can fracture if excessive force is employed during the elevation of the third molar. The tooth lies between the buccal cortical plate, which is thick, and the thin lingual cortical plate (Fig. 14.6). In most instances, the thickness of the lingual plate may be less than $1 \mathrm{~mm}$, and the tooth may get displaced into the lingual pouch if untoward force is applied.

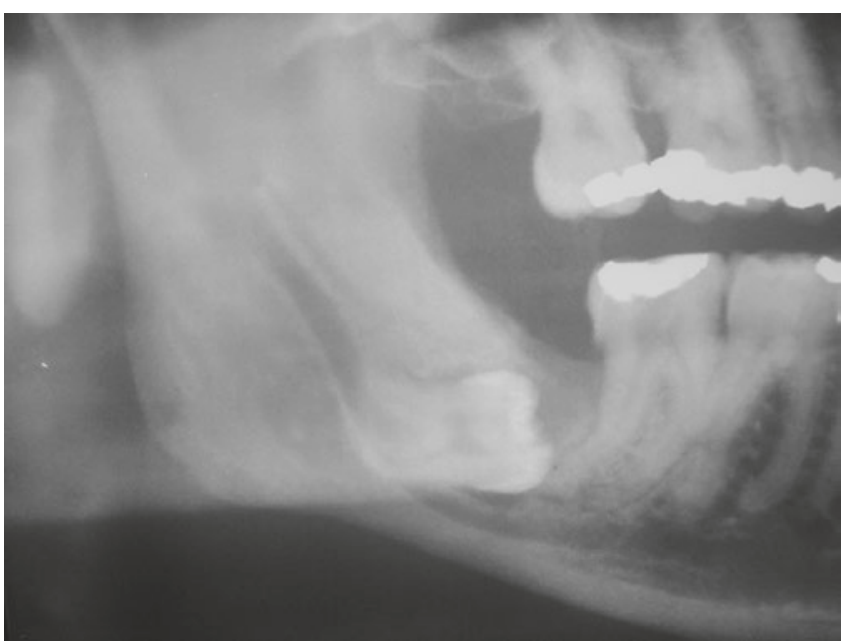

(C)Association of Oral and Maxillofacial Surgeons of India

Fig. 14.7 Radiograph showing the proximity of impacted third molar roots to the mandibular canal

\subsubsection{Neurovascular Bundle}

The mandibular canal lies beside or below the third molar roots. Usually, the canal lies slightly buccal and apical to the third molar roots, but this varies frequently (Fig. 14.7). The canal contains the inferior alveolar neurovascular bundle including the artery, vein, and nerve within a sheath of fascia. The third molar roots may sometimes be indented by the canal, but actual penetration is rare. In these cases, attempting to elevate fractured root tips may result in the displacement of the tips into the mandibular canal. If the canal vessels get injured by instruments or forceful intrusion of the tooth roots, profuse hemorrhage may result.

\subsubsection{Retromolar Triangle}

This is a depressed roughened area behind the third molar bounded by the buccal and lingual alveolar ridge crests. The retromolar fossa is a shallow depression that occurs just lateral to the retromolar triangle. Mandibular vessel branches may emerge at the fossa or triangle and can be injured during surgical exposure of the third molar region if the incision is not taken laterally. This can result in brisk hemorrhage (Fig. 14.8).

\subsubsection{Facial Artery and Vein}

The facial artery and anterior facial vein are related to the mandibular body, anterior to the masseter muscle, where they cross the inferior border of the mandible. They lie below 
the second and third molar teeth and may be injured when a buccal incision is placed at an inferior level. To avoid this, it is best to start the incision at the sulcus depth and move upward toward the tooth.

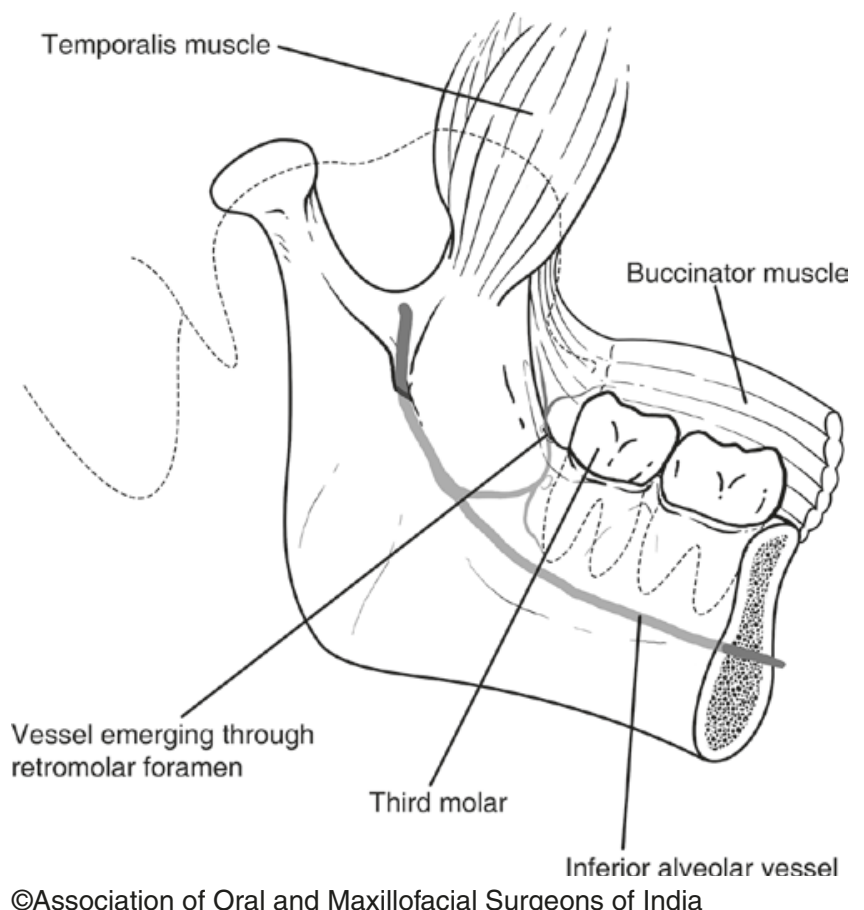

Fig. 14.8 Schematic diagram showing the retromolar vessel emerging through retromolar foramen

Fig. 14.9 Schematic diagram showing coronal section through the third molar region and the relationship of important anatomical structures

\subsubsection{Lingual Nerve}

The lingual nerve often runs below and behind the third molar, and contacts the periosteum over the lingual cortex at a sublingual level. Cardinal anatomic studies have shown the close relation of the lingual nerve to the lower third molar region $[8,9]$.

The lingual nerve usually lies $2.3 \mathrm{~mm}$ below the lingual alveolar crest, and $0.6 \mathrm{~mm}$ medial to the mandible, when viewed from a frontal plane.

Since the lingual nerve is close to the third molar, it is at risk of damage during surgical removal of the tooth. This may lead to anesthesia of the tongue in its anterior twothirds, and also loss of taste sensation in this area.

The surgeon should also be aware of the course and direction of the mylohyoid and long buccal nerve to prevent inadvertent injuries to these nerves (Fig. 14.9).

\subsubsection{Bone Trajectories of Mandible}

The bone trajectories of the mandible, referred to as grains, course in a longitudinal direction. Even though the technique of chisel and mallet has almost become obsolete, it is important to know the bone trajectories. On the buccal side, a horizontal chisel cut that is oriented parallel to the superior border may cause extensive splitting till the first molar region due to the grain direction. To prevent this, the operator must make a "vertical stop cut" (Fig. 14.10), with the bevel ori-

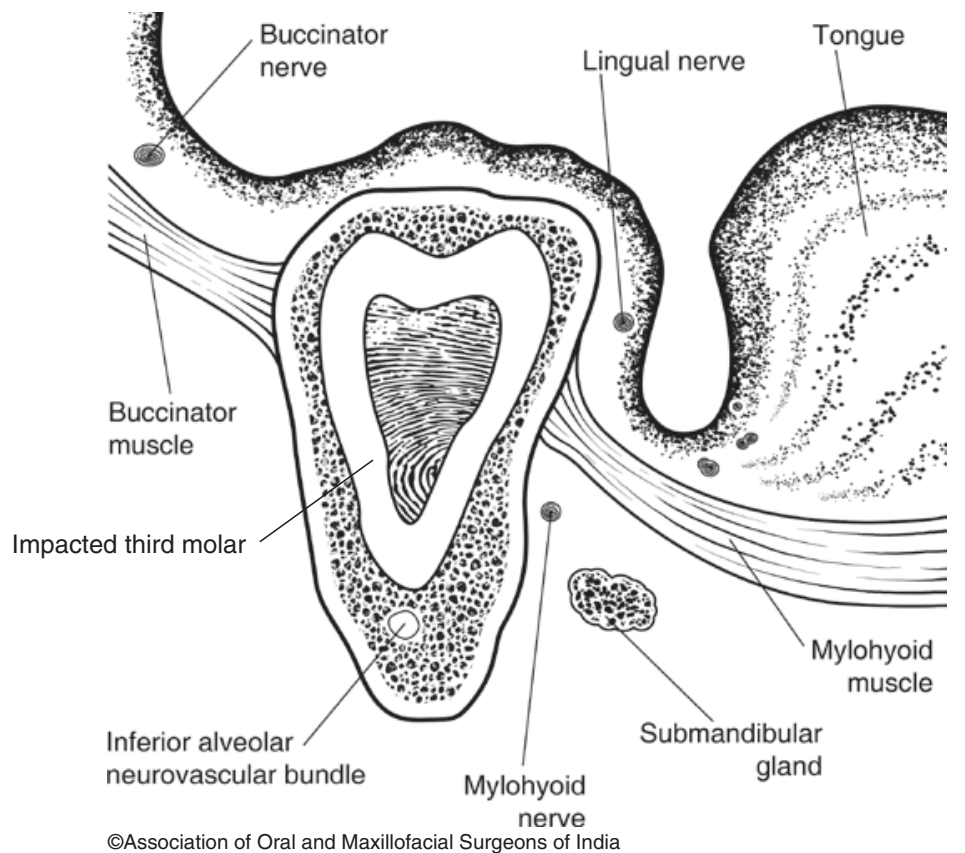




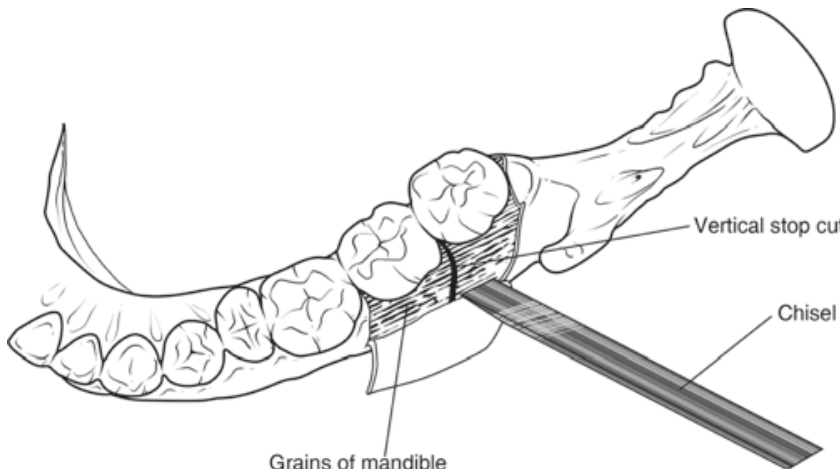

CAssociation of Oral and Maxillofacial Surgeons of India

Fig. 14.10 Bone trajectories of the mandible

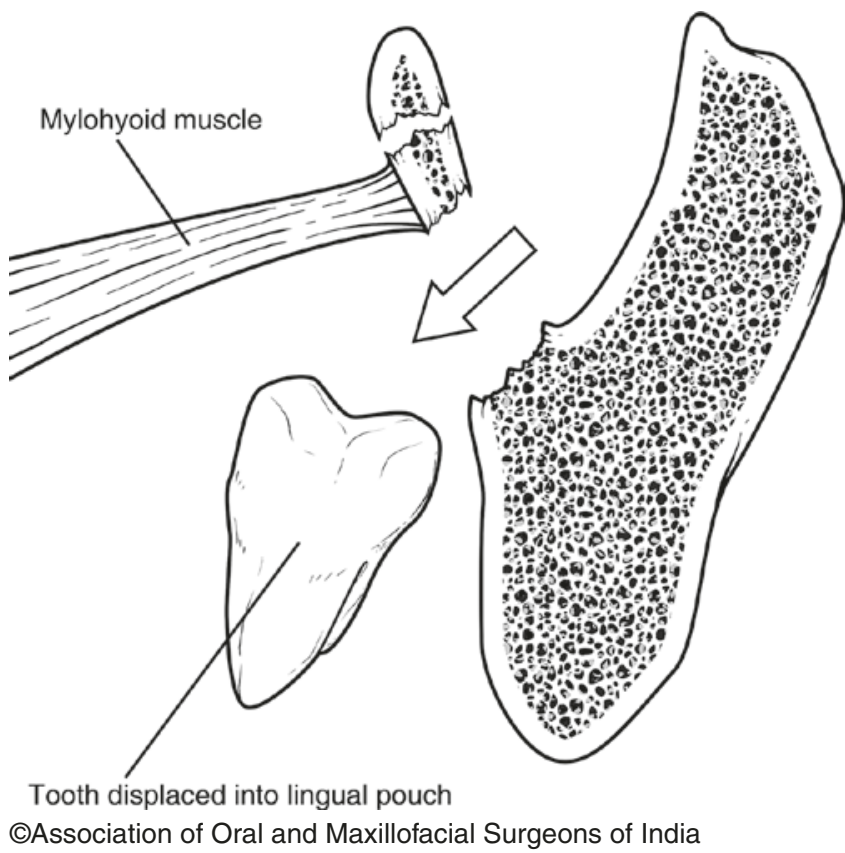

Fig. 14.11 Whole tooth displaced into lingual pouch beneath the mylohyoid muscle

ented posteriorly, just distal to the second molar. The chisel must be angulated correctly at all times to avoid fracture of mandible distal to the third molar.

\subsubsection{Lingual Plate}

Since the lingual pate is very thin, it may be perforated by the apices of lower third molar roots. If the roots are fractured, attempting to elevate may cause them to be displaced into the "lingual pouch," from where it will be difficult to retrieve. The entire tooth also may rarely be pushed into the lingual pouch (Fig. 14.11).

\subsection{Classification of Impacted Mandibular Third Molar}

To assess surgical difficulty, several classifications have been proposed, which can help formulate a treatment plan that is efficient and has minimum morbidity. Either the periapical radiograph or the Orthopantomogram is used to analyze the impacted tooth for its classification.

The most commonly used are:-

1. Angulation [10] of the impacted tooth (Fig. 14.12) (George Winter classification).

Vertical,

Mesioangular,

Horizontal,

Distoangular,

Buccoangular,

Linguoangular,

Inverted,

Unusual

2. Relationship between the impacted tooth and the anterior ramal border [11] - This assesses the amount of space present between the anterior border of the ramus and the distal wall of the second molar. This indicates the effective space available for the tooth to erupt (Fig. 14.13).

Class I-There is enough mesiodistal space between the anterior border of ramus and second molar to accommodate the third molar.

Class II-Space between anterior border of the ramus and second molar is less than the mesiodistal width of the crown of the third molar.

Class III-No mesiodistal space available and the third molar is almost completely within the ramus.

Class III impactions present greater difficulty in removal.

3. Depth of the impacted tooth and tissue type that overlies the tooth (Pell and Gregory Classification based on occlusal level of the tooth)-i.e. soft tissue, partial bony, or complete bony impaction (Fig. 14.14).

Position A-The highest point of the tooth is at the same level of the occlusal plane or above it.

Position $B$-The highest point of the tooth is above the cervical line of the second molar but below the occlusal plane.

Position $C$-The highest point of the tooth is well below the cervical line of the second molar (Figs. 14.15 and 14.16).

4. Type of tissue that lies over the tooth-i.e. soft tissue, partial bony, or complete bony impaction.

5. Level of Eruption

(a) Erupted.

(b) Partially erupted.

(c) Unerupted. 


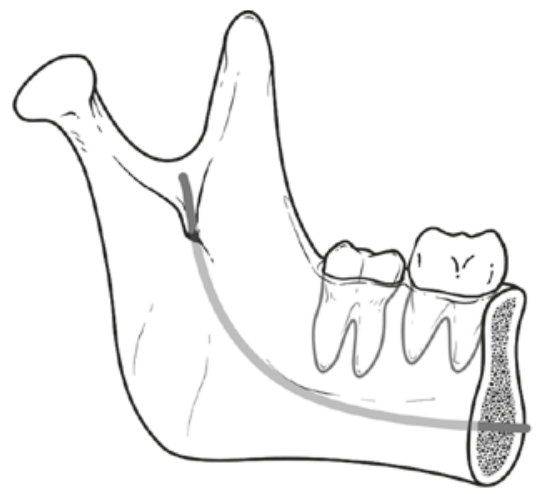

Vertical

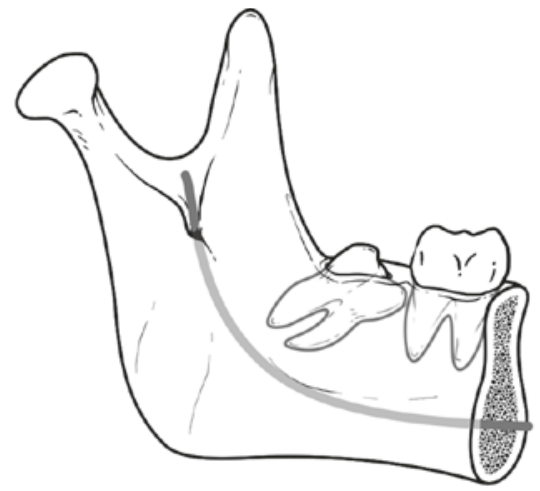

Mesioangular
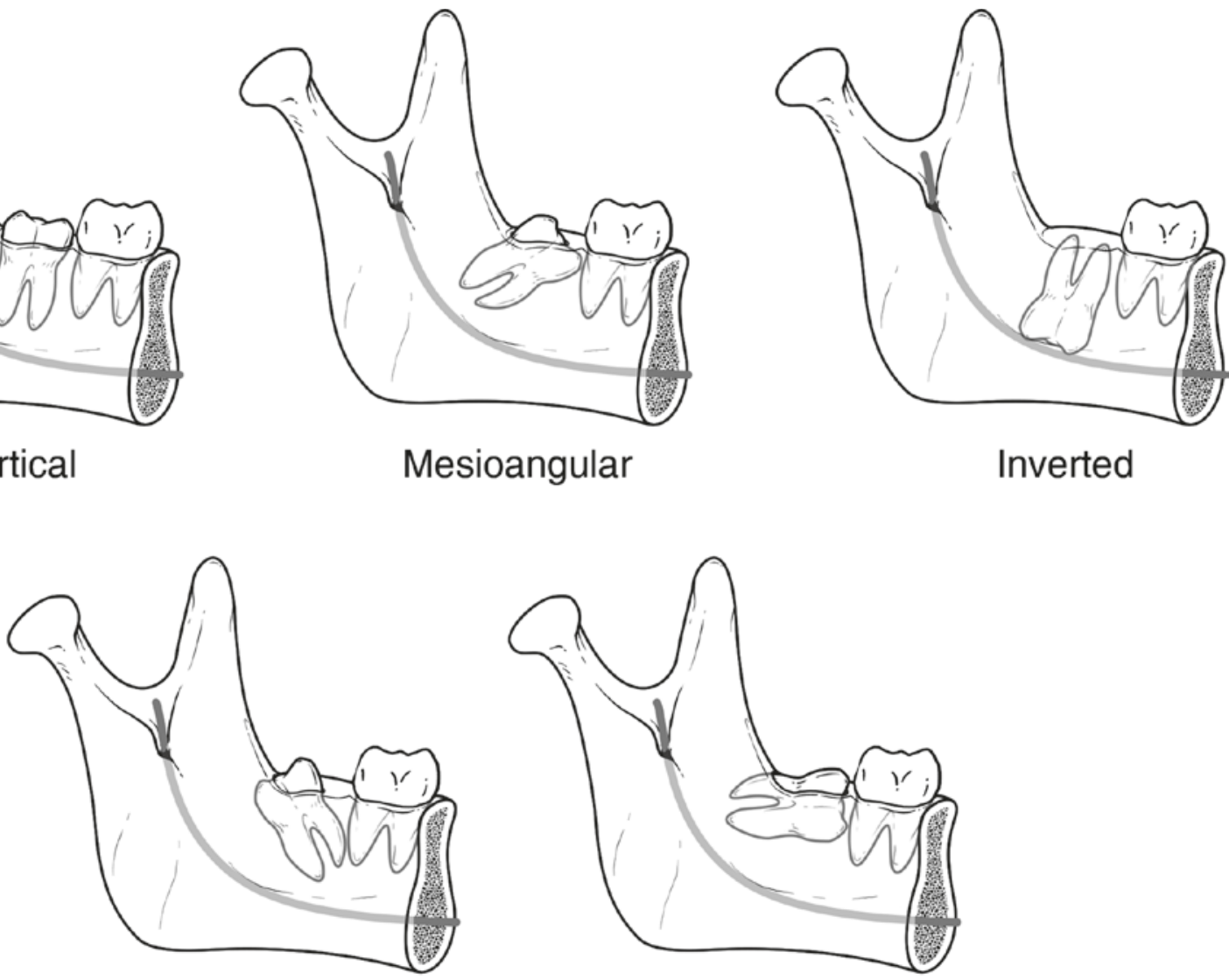

Distoangular

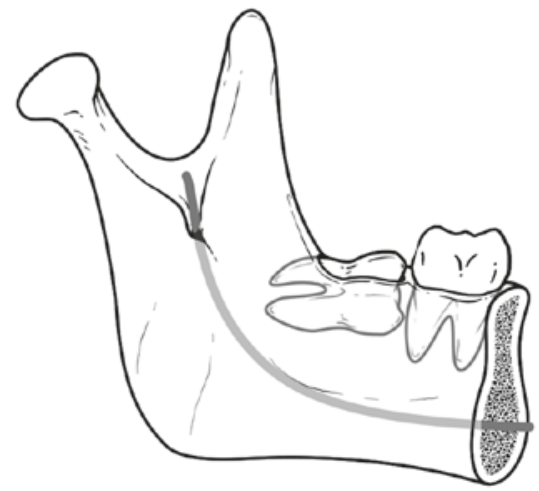

Horizontal

(C)Association of Oral and Maxillofacial Surgeons of India

Fig. 14.12 Classification based on angulation of tooth (Winter's classification)

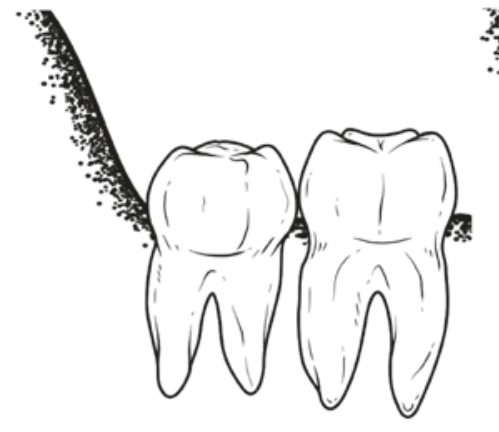

Class I

(Association of Oral and Maxillofacial Surgeons of India

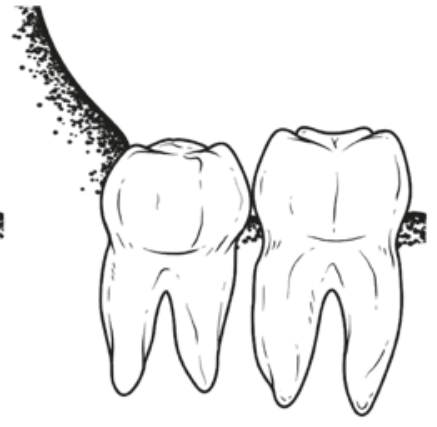

Class II

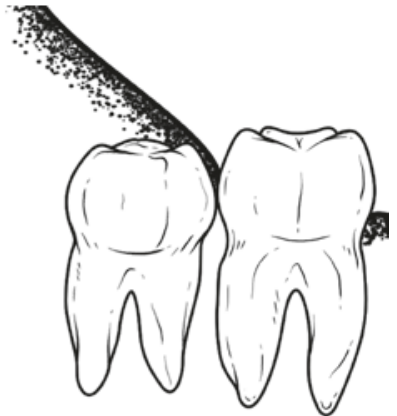

Class III

Fig. 14.13 Pell and Gregory Classification based on relationship to the anterior border of ramus 


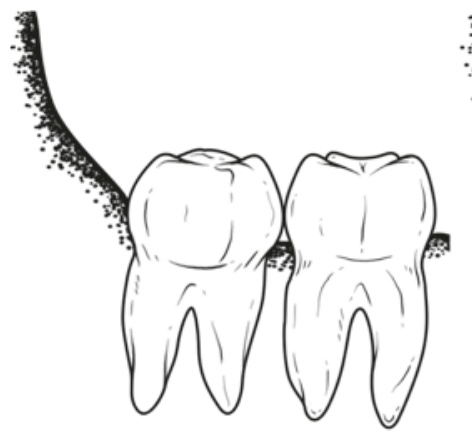

Position A

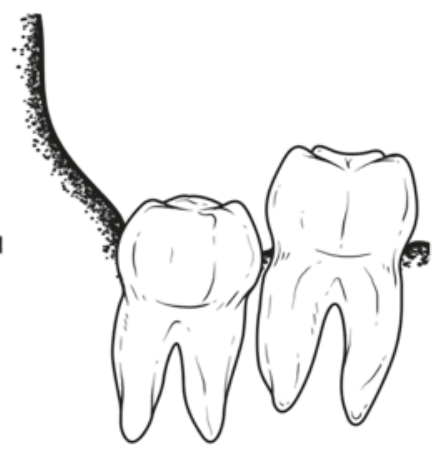

Position B

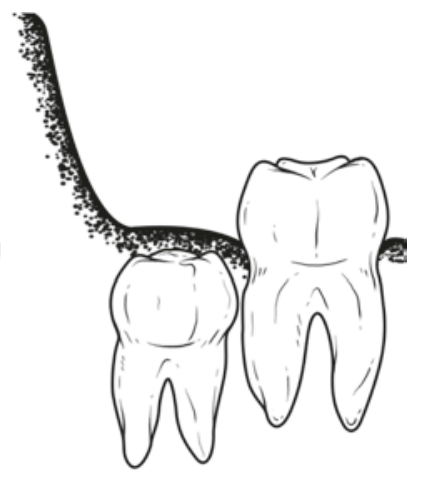

Position C

CAssociation of Oral and Maxillofacial Surgeons of India

Fig. 14.14 Pell and Gregory Classification based on relationship to the occlusal plane of the impacted tooth to that of the second molar

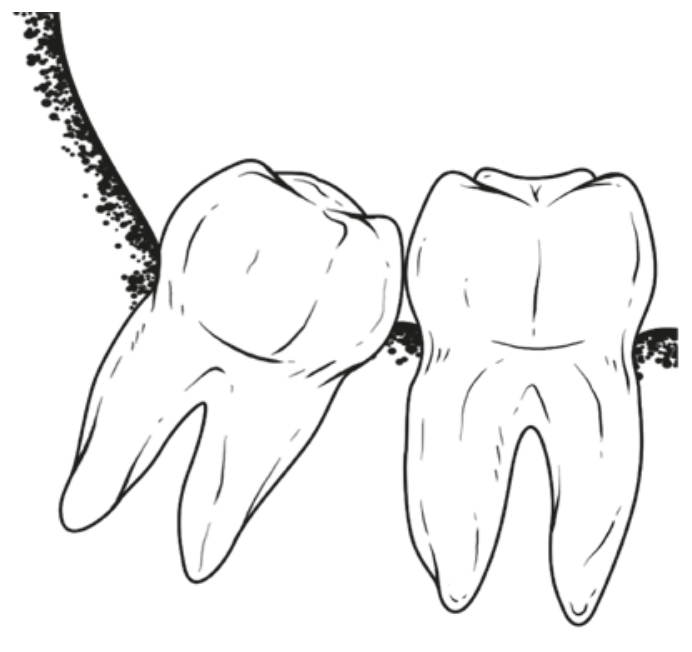

a

CAssociation of Oral and Maxillofacial Surgeons of India

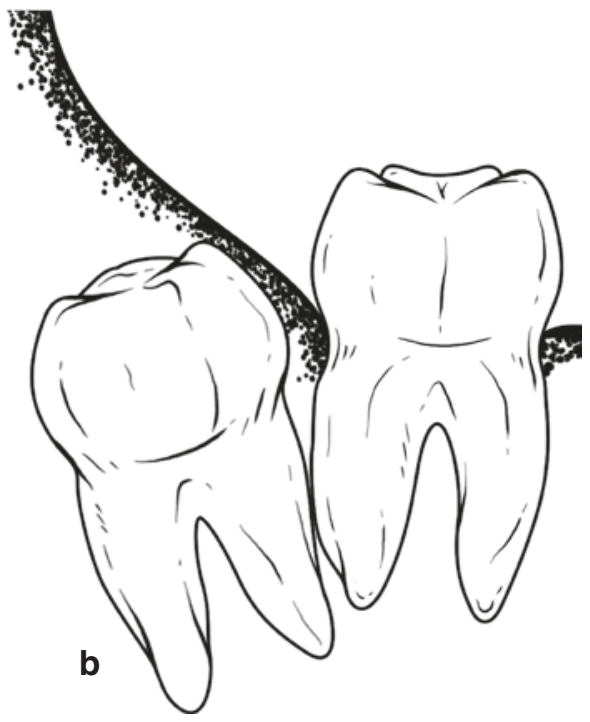

Fig. 14.15 Examples of impaction showing combination of angulation of tooth, relationship to anterior border of ramus and depth of impaction. (a) Mesioangular impaction in Class I ramus relation and Position
A depth—an impacted tooth easy for removal. (b) Distoangular impaction in Class III ramus relation and Position B depth-an impacted tooth difficult for removal 


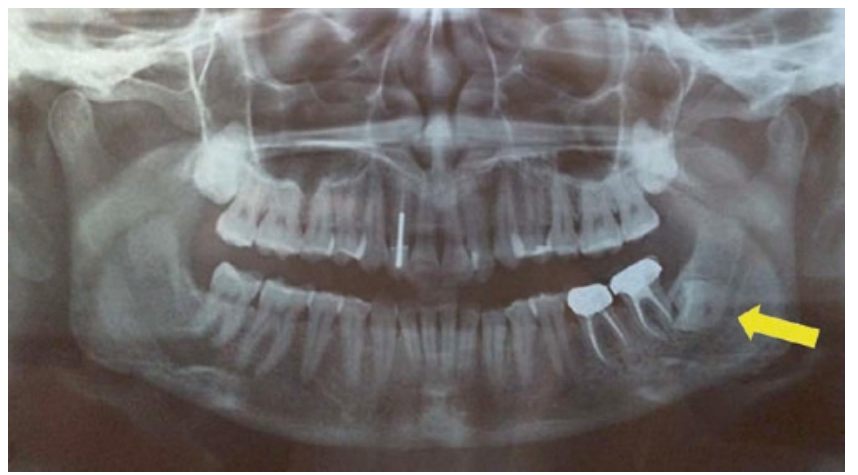

CAssociation of Oral and Maxillofacial Surgeons of India

Fig. 14.16 OPG showing impacted mandibular third molar displaying only crown of the tooth with no roots visible (yellow arrow) with close proximity to inferior alveolar canal in a 52-year-old male. Surgical removal of 38 was attempted without taking CT or CBCT. During surgery, there was accidental fracture of left angle for which internal fixation has to be done. Note impacted 18 and 28

\subsection{Preoperative Planning}

Presence of an impacted third molar must be diagnosed systematically using the patient's chief complaint and history, clinical examination, and appropriate investigations.

The impacted third molar must be evaluated both clinically and radiographically prior to surgery for successful and speedy removal. Ideally, a periapical radiograph must be taken and an OPG must be added if the intraoral radiograph does not provide enough information about the tooth or adjacent structures.

Manuel et al. has [12] developed a simple format for evaluation of third molar impactions.

This comprehensive format is ideal for residents in oral surgery during their learning years. Using this format, third molar impactions may be analyzed, and difficulty level may be assessed and anticipated. Residents can judge problems that they may encounter during the procedure and can evaluate the patient better postoperatively.

\subsubsection{Clinical Examination}

This includes taking the patient's history, clinical examination extraorally and intraorally.

\section{History taking}

Complaints of the patient-Impacted teeth are usually asymptomatic and patients are aware of their existence only when told by the dental practitioner. Symptoms, if any, are usually due to acute or chronic pericoronitis, or due to acute pulpitis secondary to dental caries.

\section{Extraoral examination}

The clinician must examine the face and neck for redness and swelling related to infection. The lower lip is tested for anesthesia or paresthesia. The regional lymph nodes must be assessed by palpation for any tenderness or enlargement.

3. Intraoral examination-The following points are noted:

(a) Mouth opening-The ability of the patient to open the mouth is analyzed, and any trismus, fibrosis, or hypermobility of the joint is noted. The size of the mouth (microsomia/macrosomia) is also checked. Third molar access may be restricted if the mandible is retrognathic, while a prognathic mandible offers good access.

(b) General examination of oral cavity- oral mucosa, teeth, and oral hygiene.

(c) Examination of the third molar area for signs of pericoronitis and state of eruption of the tooth.

(d) Condition of the impacted tooth- presence of caries, dental fillings, and internal resorption (which may resemble caries). The angle of the tooth and locking beneath second molar must be noted and confirmed with appropriate radiographs.

(e) Condition of first and second molars-presence of caries, fillings, or crowns; root canal treatment may put the second molar at risk of fracture and the patient must be warned of this. Distal periodontal pocketing, root resorption, and absence of the second molar must also be noted.

(f) Space present between the second molar distal surface and the ascending ramus: A small distance makes access difficult, and a large distance makes the tooth more accessible. For maxillary teeth, the distance between the second molar and tuberosity must be considered. Access can also be decreased by distal tilting of second molar.

(g) Adjacent bone may develop infection, which can spread along the mesial surface of the tooth and affect the second molar, which would then require extraction. Infection/osteomyelitis can spread to the ramus in the case of distoangular impacted third molars, through recurrent submasseteric abscesses in this region.

(h) Systemic skeletal diseases may cause pathological complications which should be noted. For instance, conditions such as osteogenesis imperfecta and osteosclerosis may cause fractures during the procedure. In acromegaly, the mandibular bone is massive which makes the procedure difficult because the mandible consists of massive bone. In Paget's disease also tooth removal is difficult as the bone is affected by resorption and repair. 
(i) Presence of cysts and tumors-The impacted tooth may be associated with eruption cysts or large odontogenic cysts can occur in relation to impacted tooth. By and large, they cause displacement of the tooth. Benign and malignant tumors such as ameloblastoma may also be found involving the tooth. Odontomes may also be present in relation to the third molar.

\subsubsection{Radiography of Impacted Mandibular Third Molar}

Any factor that increases the difficulty of third molar removal can be analyzed from the preoperative radiograph.

The following radiographs may be used for analysis:

1. Intraoral periapical radiograph.

2. Occlusal X-ray of mandible.

3. Lateral oblique view of mandible.

4. Panoramic radiograph (Orthopantomogram).

An essential criterion for a good film is that the buccal and lingual cusps of the second molar must be superimposed on each other in the same vertical and horizontal plane. This appearance of the second molar is referred to as 'enamel cap'.

1. Periapical radiograph: The radiograph should include the entire third molar tooth along with the investing bone. It should also show the anterior border of ramus, the second molar, and the inferior alveolar canal.

2. Lateral oblique view of the mandible: This radiograph is inevitably distorted because the opposite side of the mandible is rotated away from the beam during film exposure. Therefore, it is inferior to the periapical X-ray, but it has its use in certain clinical situations:

- When periapical X-ray cannot be taken due to trismus or retching.

- To provide supplementary information like height of mandible in the region of the third molar, or bone height beneath a deeply buried tooth. The latter is useful to assess the risk of pathological fracture in thin mandible, or in cases of cysts or tumors.

Since the introduction of OPG, the use of lateral oblique view is limited and is only considered when an OPG is unavailable.

3. Orthopantomogram $(O P G)$ : This provides the same information as the lateral oblique view, with less distortion. The OPG is now used routinely to precisely the locate impacted teeth.

\subsubsection{Interpretation of Periapical X-ray}

The following points must be noted in the periapical radiograph:-
(a) Access.
(b) Depth and position of the tooth
(c) Root pattern of impacted tooth.
(d) Shape of crown.
(e) Texture of investing bone.
(f) Relation to inferior alveolar canal.
(g) Root pattern and position of second molar.

(a) Access-By observing the inclination of the radioopaque line that is formed by the external oblique ridge, the ease of access can be ascertained. A vertical line implies poor access and a horizontal line, good access.

(b) Position and depth of impacted tooth-These can be evaluated using Winter's technique of WAR lines (described by George Winter). WAR refers to three imaginary lines drawn on the radiograph, namely, the "white," "amber," and "red" lines (Fig. 14.17a, b).

The first line or "white" line extends across the occlusal cusp tips of the erupted mandibular molars and is drawn distally over the third molar region. This line indicates the axial inclination of the impacted tooth. For example, the white line is parallel to the occlusal surface of the third molar if the tooth is vertically impacted, whereas, the "white" line converges with the occlusal surface in front of the tooth in distoangular impactions.

The "white" line also indicates the depth of the tooth as compared to the erupted second molar.

The second "amber" line extends from the bone surface distal to the third molar and is drawn to the interdental septum crest between the first and second molar. When drawing this line, it must be clearly differentiated from the external oblique ridge shadow, which can lie above and in front of the posterior end of the "amber" line. The posterior end is the shadow cast by the bone in the retromolar fossa and not the external oblique ridge. The "amber" line shows the margin of the alveolar bone enclosing the tooth. Hence, when soft tissues are reflected, the portion of the visible tooth will be the part that was lying above and in front of the "amber" line in the radiograph. The rest of the tooth will be covered by bone.

The third line or "red" line assesses how deep the impacted tooth is within the mandible. This is drawn by dropping a perpendicular from the "amber" line to the point at which the elevator will be applied to elevate the tooth (an imaginary point). This point usually lies on the mesial surface of the impacted tooth, at the cemen- 


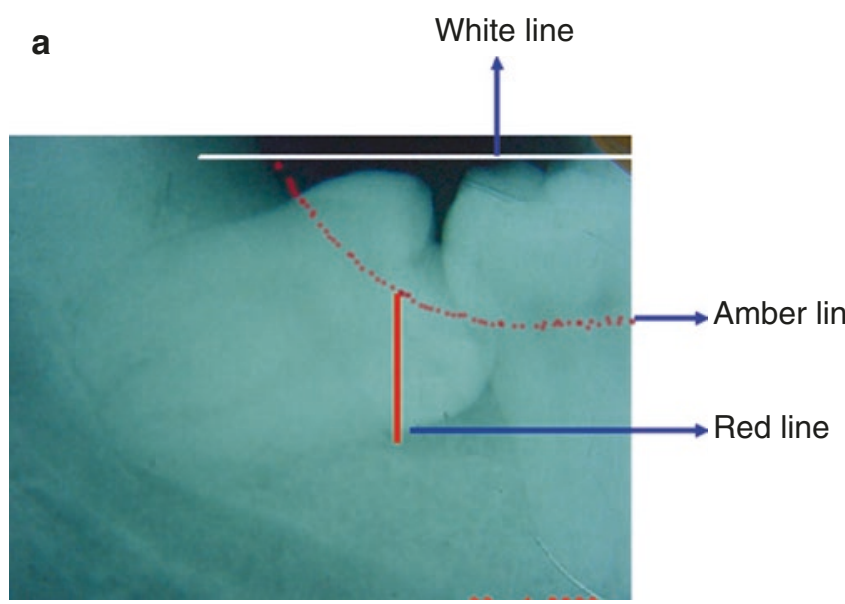

CAssociation of Oral and Maxillofacial Surgeons of India

Fig. 14.17 (a) White, amber, and red lines (Winter's WAR lines) marked in the periapical X-ray. (b) WAR lines drawn on a distoangularly impacted mandibular third molar. Note that in distoangular impac-

toenamel junction, except in the case of distoangular impactions. The longer the red line, the more deep the tooth is impacted, and the surgical procedure will be more difficult.

(c) Root pattern of impacted tooth-The number, shape, and curvature of roots are noted. Hypercementosis is noted if present. If the root apex takes a sharp bend toward the X-ray beam, it may appear blunt and short on the image. This finding should therefore be investigated in more detail.

The type of root morphology dictates the difficulty of the surgical procedure. If root development is limited, it can result in a "rolling" tooth, which is challenging to remove.

(d) Shape of crown-If the tooth has prominent cusps, or large, square crowns, the difficulty increases as compared to small crowns and flat cusps. The size and shape of the crown of third molar is particularly important with regard to the "line of withdrawal." Sometimes, the path of crown removal can be obstructed by the second molar crown (Fig. 14.18).

In these cases, the cusp of the third molar appears to be superimposed on the distal surface of the second molar in the radiograph. If elevation is attempted by applying force on the mesial surface of the impacted tooth, the second molar may get displaced from the socket, and there is a risk of mandible fracture. The risk is especially high for second molars with conical roots. In such cases, sectioning the third molar is advisable.

(e) Texture of the investing bone-As age advances, the bone undergoes sclerosis and becomes less elastic. The bone texture can be analyzed by visualizing the size of the cancellous spaces and the bone density. Bone that has large spaces and fine structure is generally elastic.

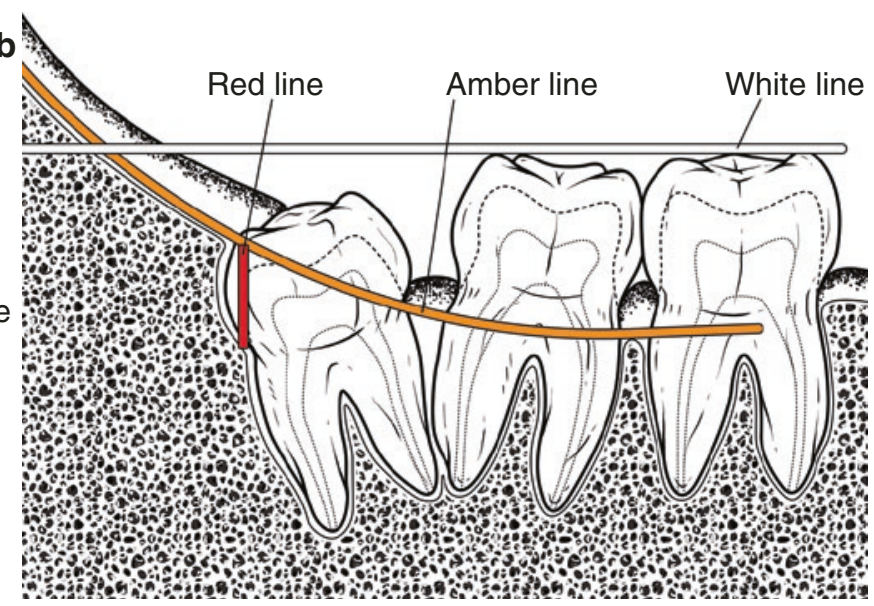

tions, the perpendicular "red" line should be dropped to the cementoenamel junction on the distal side of the impacted tooth and not on the mesial side as in other angulations

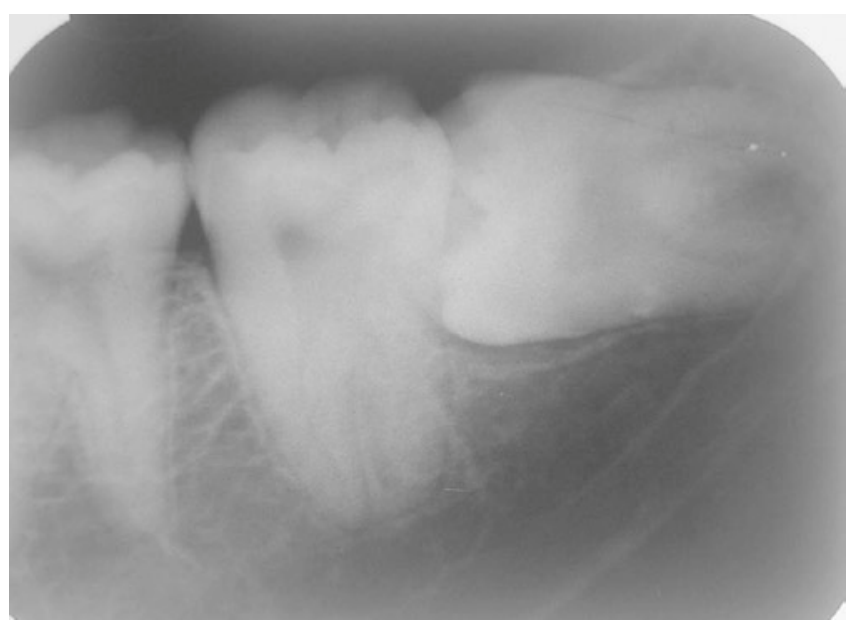

(CAssociation of Oral and Maxillofacial Surgeons of India

Fig. 14.18 "Locking of the crown" of impacted third molar by the second molar. Note that the cusp of third molar is superimposed upon the distal surface of second molar

On the contrary, sclerotic bone has small spaces and dense bone structure. Dense bone does not expand easily during luxation and more bone removal may be required.

(f) Inferior alveolar canal-Although radiographs often show the canal crossing the third molar roots, this is usually due to superimposition. Sometimes, however, this may indicate grooving or perforation of the root.. The classical papers by Howe and Poynton [13] and Rood and Shehab [14] have given predictable signs to assess the relationship between the nerve and the third molar roots.

1. If there is a band of reduced radio-opacity that crosses the roots, and this band coincides with the outline of the inferior alveolar canal, this is a sign that the tooth 
root may be grooved by the canal. This sign reflects the lesser amount of tooth structure lying between the X-ray source and the film.

2. The roof and floor of the canals are formed of compact bone, which is indicated by continuous, parallel radio-opaque lines. If the lines lose continuity, it is an indication that the root is grooved by the inferior alveolar canal. These grooves usually form on the lingual side of the roots.

3. In cases where the radiolucent band crosses the apex of the root and if only the upper white line is broken, a notching of the root is present.

4. Characteristic narrowing of the radiolucent band with loss of white lines is suggestive of perforation of the root by the inferior alveolar canal.

The following signs have been demonstrated to be associated with a significantly increased risk of nerve injury during third molar surgery: (Fig. 14.19a, b, c)

- Diversion of the inferior alveolar canal (IAC).

- Darkening of the root where crossed by the canal.

- Interruption of the white lines of the canal.

In the presence of any of the above findings, great care should be taken in surgical exploration and the decision to treat is carefully reviewed. If on the initial panoramic radiograph there is an evidence of a close relationship between the roots of the lower third molar and the IAC, a second radiograph should be taken using different projection geometry.

If the third molar is found to be in close relationship with the inferior alveolar canal, the patient should be informed in advance regarding the likelihood of impairment of labial sensation following the surgical removal of the tooth. This should be recorded in the case record and in the consent form. In such cases, authors have recommended coronectomy (partial tooth removal, intentional root retention, partial odontectomy) as an option. However, this technique cannot be considered foolproof and long-term studies are required to know the success of coronectomies $[15,16]$

(g) Position, root pattern, and nature of crown of second molar.

The space between the distal surface of the second molar and the mesial surface of the impacted third molar has an effect on the ease of removal of the third molar. The closer the third molar is to the second molar, the more challenging the surgery becomes. If the long axis of the second molar is tilted distally, it is more difficult to remove.

Cone beam computed tomography (CBCT) (Fig. 14.20a, $\mathrm{b}, \mathrm{c})$ CBCT is now available for dental use and offers low dose imaging in multiple planes.

Using this modality, accurate three-dimensional imaging can be done to determine the relationship between the roots of the third molar and the inferior alveolar nerve (IAN). The recent recommendation is that when the OPG suggests a close relationship between the roots of the lower third molar and IAN, cone beam CT scanning should be advised [17-19]. The effective dose from CBCT is comparatively less than the conventional CT scan and also at a lower expense. a
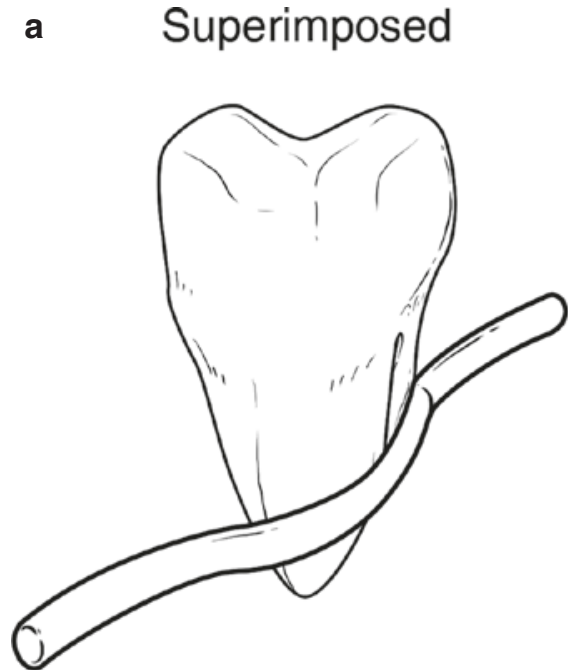

(C)Association of Oral and Maxillofacial Surgeons of India b Grooved

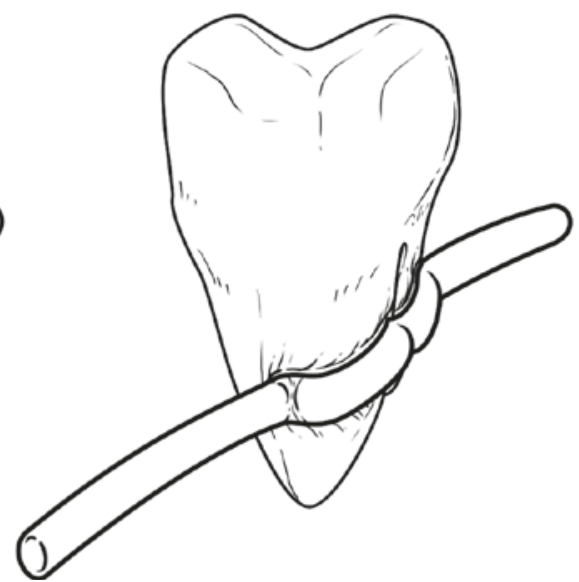

C

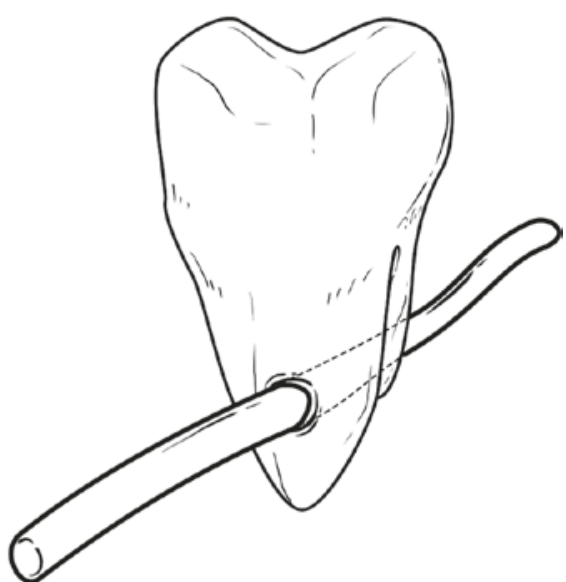

Fig. 14.19 (a-c) Radiographic relationship of third molar root to inferior alveolar nerve (a) Cortical outline of the canal is intact. This probably represents superimposition only. (b) There is loss of cortical outline of the nerve canal. The nerve may be grooving the tooth. (c)
There is loss of cortical outline as well as narrowing and deviation of the nerve canal, denoting an intimate relationship of the nerve with the tooth and possibly perforation of the tooth roots by the nerve 


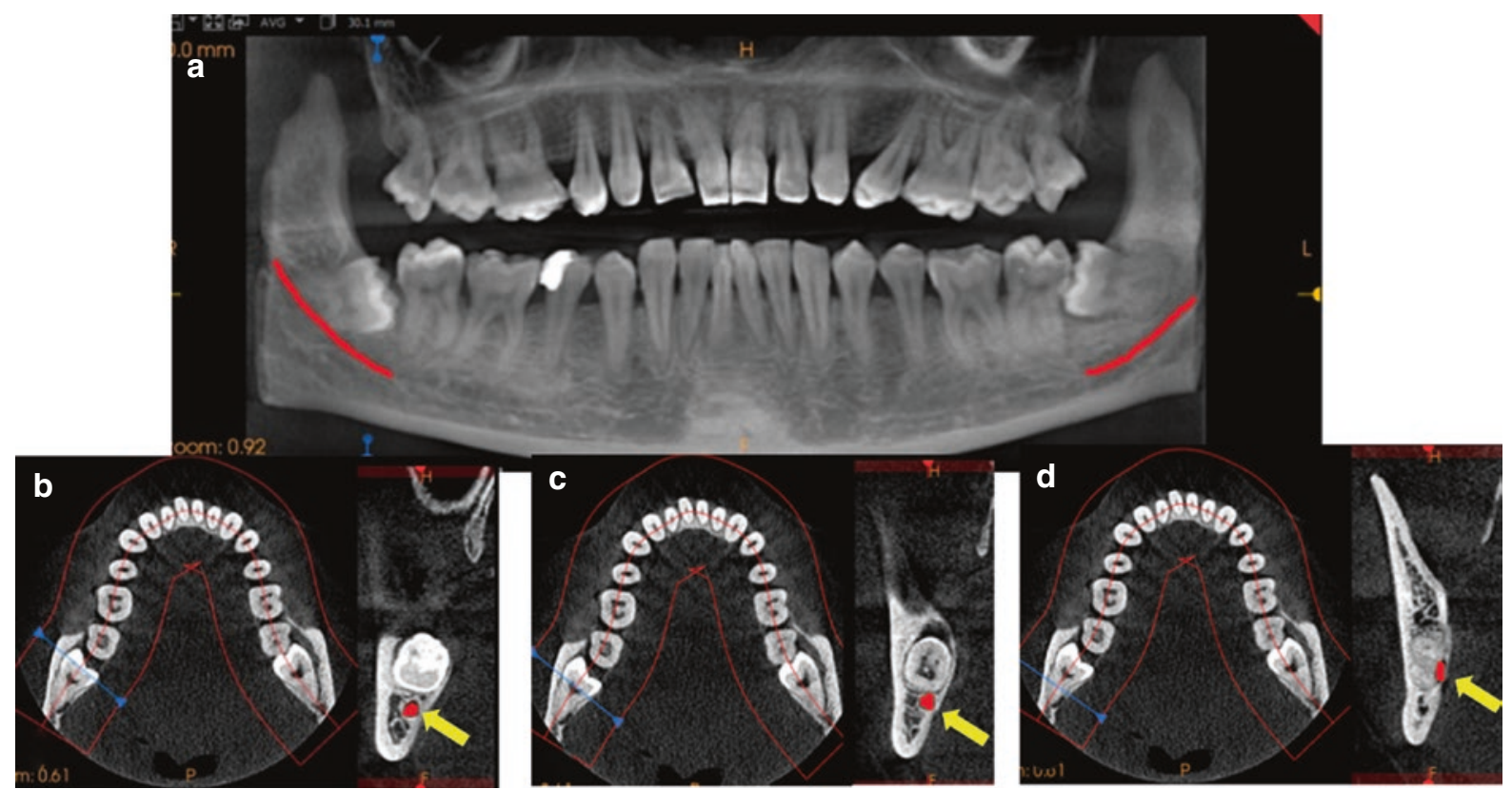

(C)Association of Oral and Maxillofacial Surgeons of India

Fig. 14.20 (a) CBCT Panoramic view showing the relationship of 38 and 48 to the inferior alveolar canal (IAC) [red line]. The changing relationship of the IAC to 48 can be noted in (b-d). Relationship of IAC

\subsubsection{Lingual Nerve Protection and Injury}

Locating the lingual nerve clinically and by imaging is more challenging. Lingual nerve injury although less common than inferior alveolar nerve (IAN) injury is often more unbearable to the patient. Patients find it difficult to tolerate lingual nerve damage, including loss of taste and sensation of tongue, as compared to IAN damage. Unlike the IAN, the lingual nerve is not usually imaged prior to third molar surgery. In cases where distal, distolingual, and lingual bone is to be removed, technique of raising the lingual flap and protecting the lingual nerve by a broad smooth lingual flap retractor in a subperiosteal plane has been advocated by certain authors and this technique is followed in certain parts of the world. Again, there are conflicting reports where the lingual flap retraction itself has caused an increase incidence of lingual nerve paresthesia [8, 20-22].

The incidence of IAN involvement 1-7 days after surgery is around $1-5 \%$ and the incidence of lingual nerve involvement one day after surgery (excluding the use of lingual flap elevation) varies from 0.4 to $1.5 \%$ [23, 24].

\subsubsection{Preoperative Evaluation of Difficulty of Removal}

Various techniques have been suggested for the preoperative evaluation of difficulty, but these have often been of limited (yellow arrow) at coronal level (b), at cervical level (c), and at apical one third level $(\mathbf{d})$

value. Pederson [25] recommended a scale to evaluate the difficulty index.

Although the Pederson scale can be used for predicting operative difficulty, it is not extensively used [26] because it does not take various other relevant factors into account, such as bone density, flexibility of the cheek, and mouth opening.

Surgical removal impacted third molar becomes difficult with the following factors:-

1. Unfavorable root morphology- Excessive curvature, divergent roots, hypercementosis, proximity to canal

2. Third molar crown is locked beneath the second molar.

3. Condition of the impacted tooth (Carious or with filling).

4. Condition of second molar- carious or with filling/ crown or any resorption.

5. Sclerosis of adjacent bone.

6. Mouth opening - If the patient has a small commissure, or trismus, access becomes limited.

7. Large follicular sac around the crown-makes procedure easier.

8. Width of the periodontal membrane-In patients past middle age, the space containing it is much smaller than in young patients. This makes removal difficult.

9. Existing fracture of the jaw.

10. Local or systemic pathologic conditions.

11. Age of the patient. 
Surgical removal of impacted teeth may be easier in younger patients because of incompletely formed roots, large follicular space, incompletely formed roots separated from inferior alveolar canal, and greater elasticity of bone. In young patients, the bone texture is usually soft and resilient, but in older adults, the bone becomes progressively more dense, hard, and brittle. Therefore, the extraction of a partially erupted/impacted tooth in an elderly adult with sclerotic bone may cause great difficulty. While a tooth with adverse root morphology in soft, resilient bone of a young adult can be elevated expeditiously.

To summarize, the difficulty of the surgical procedure is dictated by 3 major factors:- (1) Depth of impaction (2) Type of overlying tissues, and (3) Age of the patient.

As a general rule, the more difficult and time-consuming the surgical procedure is, the more difficult and protracted is the postoperative recovery period.

\subsection{Operative Procedure}

Any standard operative plan consists of the following stages:-

1. Placement of an incision to access the region of the impacted tooth.

2. Removal of enough bone to allow for delivery.

3. Sectioning the tooth and delivering it from the socket.

4. Debridement of the surgical site.

5. Wound closure.

\subsubsection{Incision and Designing the Flap}

Envelope flap, which is commonly used, extends from the posterior margin of the impacted tooth, and runs forward till the level of the first molar. The posterior end of the incision is directed buccally along the external oblique ridge (Fig. 14.21a, b).

If greater access is required, the envelope flap will not be adequate. In such cases, a release incision is given on the anterior-most point of the incision, which creates a triangular flap (Fig. 14.22a, b). This incision must begin at a point that lies approximately $6 \mathrm{~mm}$ below the gingival margin in the buccal sulcus and then extend upward in an oblique fashion to the gingival margin. The incision ends on the margin at a point between posterior and middle thirds of the second molar.

The envelope incision has been associated with fewer complications, and healing occurs faster as compared to the triangular flap. A small artery, the buccal artery, may sometimes be encountered while placing the releasing incision, and mild bleeding can result if this is injured. If more exposure is needed, the vertical release incision can be brought forward, and placed between the second and first molar as shown in the Fig. 14.23a, b.

The incision is then continued along the cervical line of the second molar and reaches the middle of its posterior border. The incision continues in a posterior and lateral direction, along the anterior border of the ramus, depending on the exposure required. It is essential that this arm of the incision is oriented laterally, and not in a straight line, because the mandible diverges laterally. If the incision is extended straight, the knife may cause lingual nerve damage. The lateral extension will also preserve small vessels that emerge from retromolar fossa (Fig. 14.24).

a
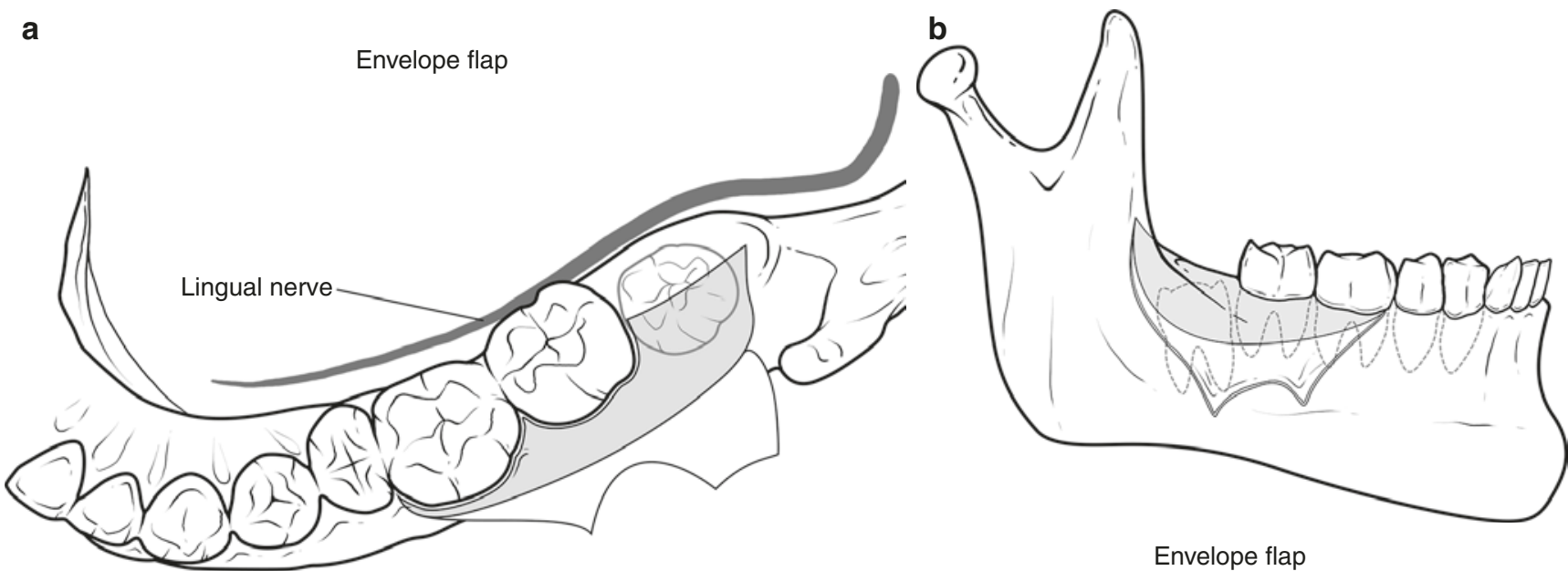

Envelope flap

CAssociation of Oral and Maxillofacial Surgeons of India

Fig. 14.21 (a, b) Envelope flap design 

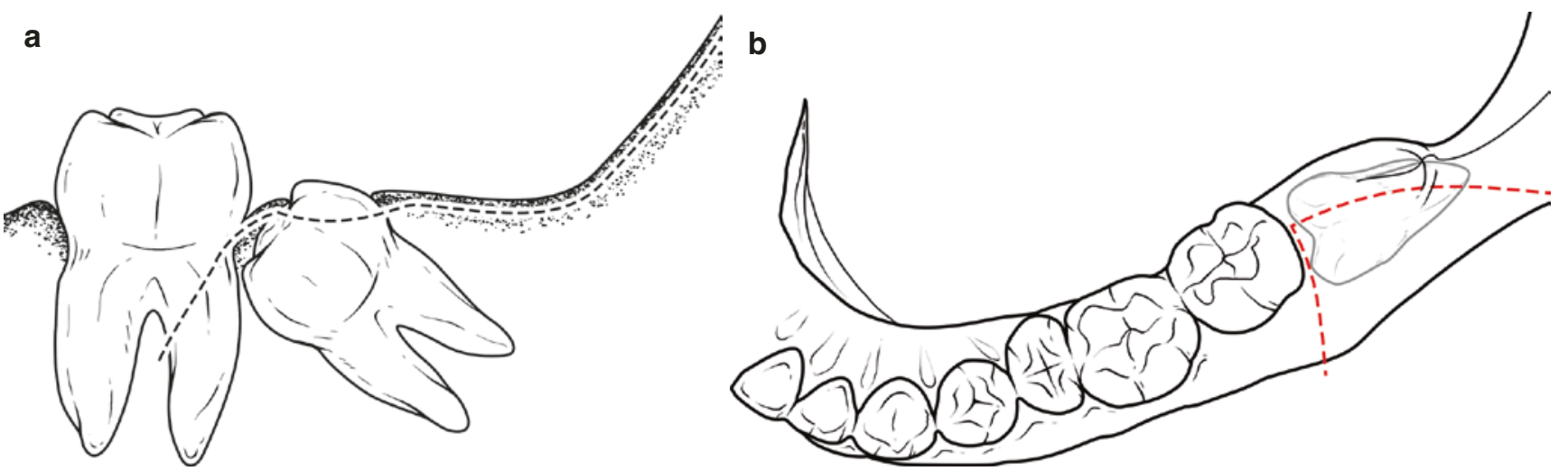

CAssociation of Oral and Maxillofacial Surgeons of India

Fig. 14.22 (a, b) Standard triangular flap with a release incision in the anterior aspect
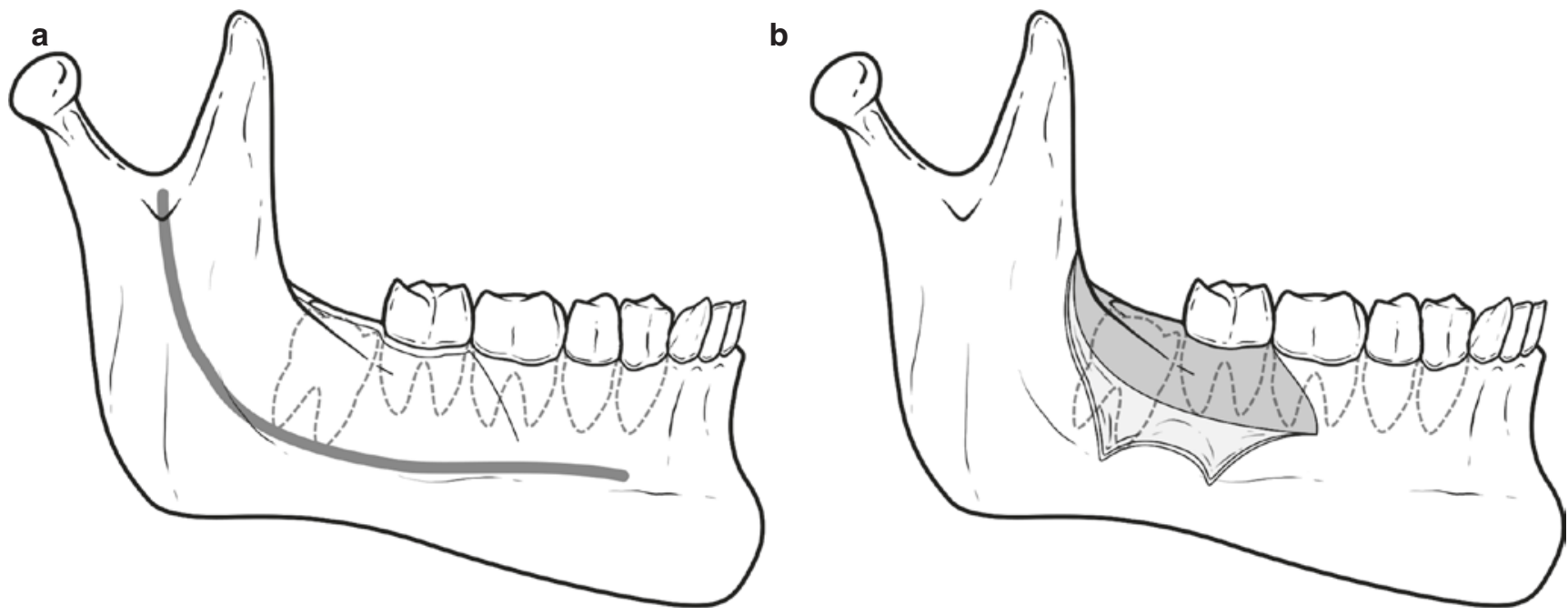

CAssociation of Oral and Maxillofacial Surgeons of India

Fig. 14.23 (a, b) Where more exposure is needed, the vertical release of the triangular flap is placed between the second and first molar

a

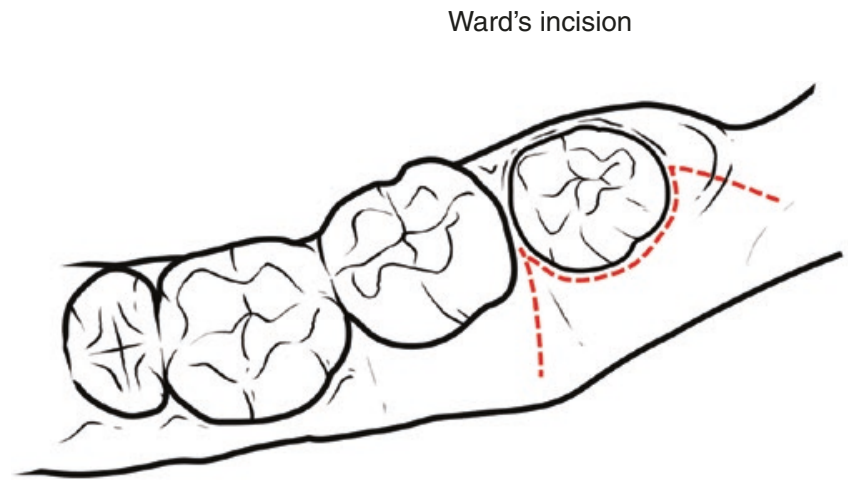

CAssociation of Oral and Maxillofacial Surgeons of India b

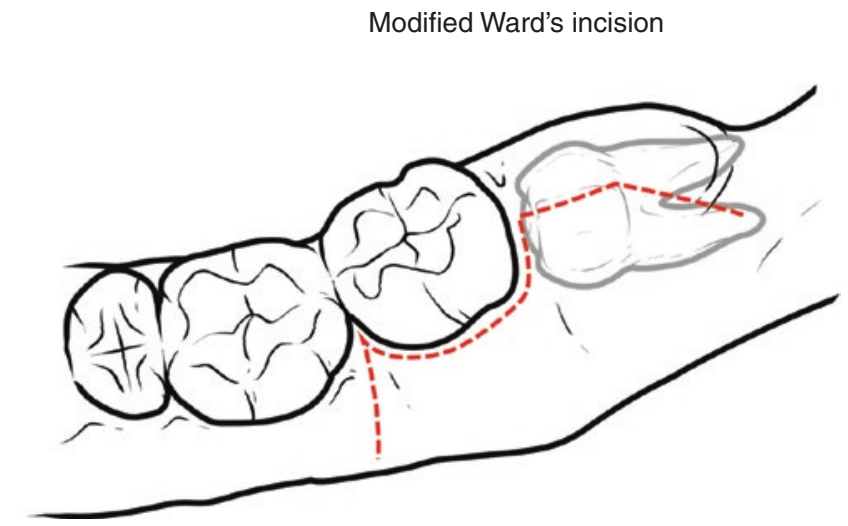

Fig. 14.24 (a) Classical Terrence Ward's incision, (b) Modified ward's incision 
The mucoperiosteal flap is then reflected laterally with a periosteal elevator. An Austin's retractor (third molar retractor) is used to hold the flap in position. The "Minnesota retractor" may also be used to hold the flap. This retractor must be placed just lateral to the external oblique ridge. Stability is achieved by resting against the lateral surface of the mandible. While holding the retractor, fingers must rest at its distal end so that the retractor can be moved laterally without blocking the vision of the operator.

The literature shows various flap designs with modifications for lower third molar impactions with each claiming its own merits [27, 28]. However, for the majority of the cases, the conventional flap designs will serve the purpose.

\subsubsection{Bone Removal}

After flap reflection, the next step is bone removal from around the impacted tooth. The amount of bone to be removed will depend on the depth of impaction. This can be done either by use of bur/rotary instruments or by chisel and mallet or ultrasonic devices/peizo surgery [29, 30] or laser devices [31]. The method used may depend on individual preference. Sufficient amount of bone must be removed, both to free the tooth from obstruction and to provide a point of application for the elevator.

The buccal cortex plays an important role in maintaining the strength of the mandible. Hence, the removal of buccal bone should be minimized, in order to prevent weakening and fracture of the mandible. The bone buccal to and distal to the impacted tooth must be removed until the cervical line of the tooth. Beyond this, bone removal must be done judiciously, in such a way that the strength of mandible is not affected, but, at the same time, the efficiency of surgery is maintained. To achieve this, a deep vertical gutter is drilled on the buccal side, and, if required on the distal side of the tooth. This "guttering method" maintains the buccal plate height, does not weaken the mandible, and at the same time, creates adequate space around the tooth to permit its free movement (Fig. 14.25).

The "Postage stamp" method of bone removal used in transalveolar extractions can also be used to remove the buccal bone, but this method may be more time-consuming. The surgical removal of an impacted tooth is basically a transalveolar extraction and all the basic principles, right from the mucoperiosteal flap design to bone removal and closure has to be followed religiously to achieve good healing.

Bone can also be removed from the mesial aspect of the impacted tooth using this method. In this region, bone removal must be extremely conservative to avoid damage to the distal aspect of the adjacent second molar. Extreme

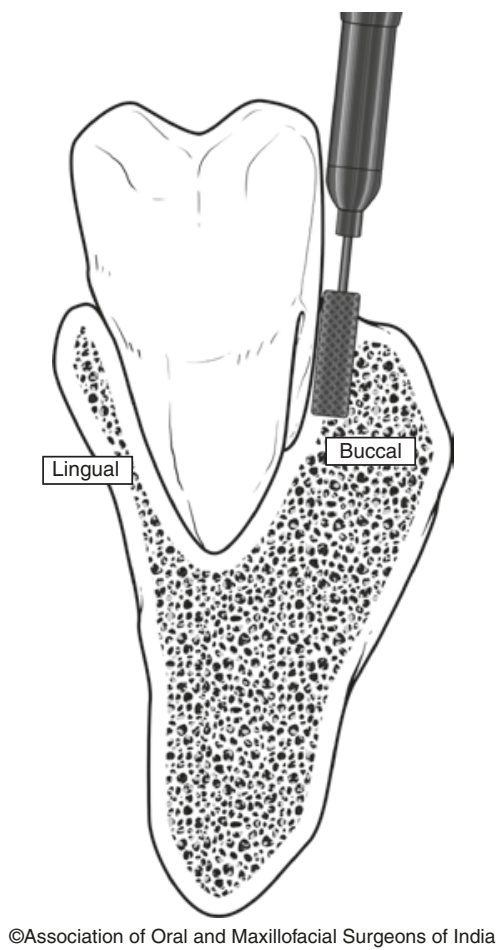

Fig. 14.25 "Guttering method"- A deep vertical gutter using bur is made alongside the buccal aspect and if required on the distal aspect of the tooth

care is taken while removing bone on the distolingual aspect, and proper retraction must be used to prevent lingual nerve damage from the bur. Due to the likelihood of damage to the lingual nerve, bone must not be removed on the lingual aspect. The commonly used burs for bone removal are the \#8 round bur and a \#703 fissure bur, although a wide variety may be used based on individual preference.

After the tooth is exposed, a point of application is created for the elevator. This allows the tooth to be displaced using only moderate force. If the tooth is resistant, further bone removal or tooth sectioning must be considered.

\subsubsection{Elevation of Tooth from the Socket}

Once bone removal is complete, tooth elevation can be attempted. Undue force should not be used for this purpose. Applying inappropriate amount of force, especially without sufficient bone removal, can cause the tooth to fracture, or can even cause fracture of the mandible. Because of the above risk, the use of instruments with high mechanical efficiency is contraindicated for third molar removal. These instruments include dental extraction forceps and cross bar 
elevators. Once the obstructing bone has been removed, only a small amount of force alone is needed to deliver the tooth. Elevators such as the Warwick James elevator (both straight and curved types) and Coupland elevator, which have lower mechanical efficiency, may be used for this purpose.

\subsubsection{Sectioning and Tooth Delivery}

If the tooth has been sufficiently exposed but is still resistant to moderate force, tooth sectioning must be considered. The tooth is sectioned into appropriate pieces for easy delivery from the socket. Sectioning of the tooth not only avoids additional bone removal, but it also reduces operating time. Tooth sectioning can be carried out using a bur, which is preferred, or a chisel. In the standard technique, sectioning is carried out using the bur at the neck of the tooth, which facilitates crown removal first, followed by the roots in a single piece. Alternatively, the tooth may be divided horizontally also. Nevertheless, in cases of divergent roots, or where the path of withdrawal is complex, the roots may have to be divided and removed separately.

The manner of sectioning of crown and root must be decided individually for each case and the standard technique need not be followed exactly (Fig. 14.26).

\subsubsection{Modifications of standard technique}

Although the principles of third molar removal remain fundamentally the same, the angulation of the tooth may dictate certain modifications. Angulation dictates the site of application of the elevator, as the path of withdrawal of the third molar should be along the line of least resistance. Therefore, the angulation, in terms of mesioangular, horizontal, vertical, and distoangular impactions must be considered.

The mesioangular impaction (Fig. 14.27a, b, c) (Video 14.1) is generally considered to be the least difficult to remove. After elevating the mucoperiosteal flap and exposure of the crown, the buccal guttering is carried out till the mesial surface of impacted tooth, to a point below the cementoenamel junction. This allows the tip of the elevator to be introduced to engage beneath the cervical cementum on the mesial side of the tooth. When the elevator is rotated, the interdental bone is used as the fulcrum and the tooth rotates distally. Thus the tooth, which had an initial mesial angulation, now occupies a vertical position. When more force is applied using the elevator, the tooth is delivered. In some instances, although the tooth becomes vertical, further movement is prevented by the distal bone. This obstruction may be relieved by one of the following methods:

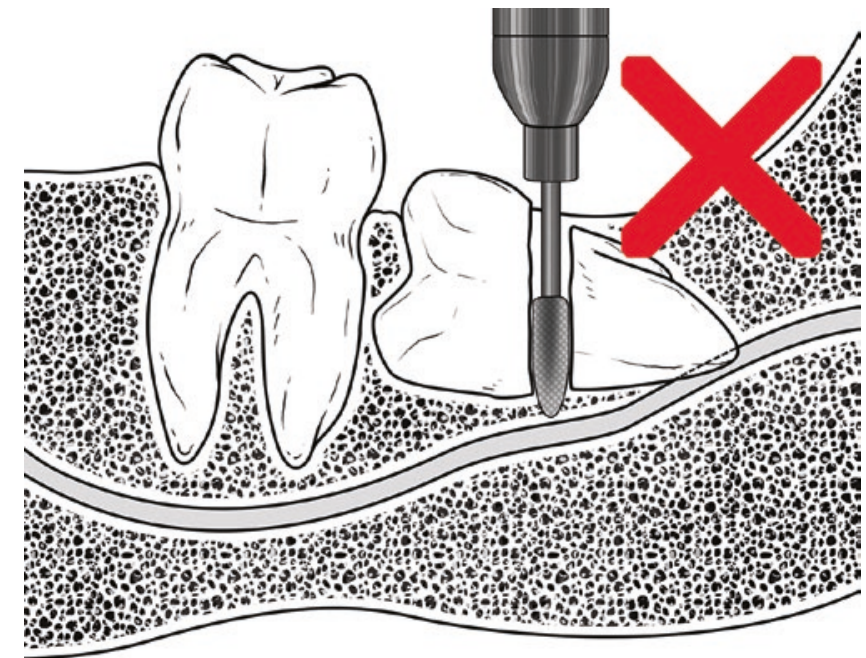

CAssociation of Oral and Maxillofacial Surgeons of India

Fig. 14.26 Possible damage to inferior alveolar neurovascular bundle if the bur is carried to the full width of the tooth inferiorly. Hence, bur is used to cut only three-fourth width of the tooth and the rest of the tooth is separated using a suitable instrument

(a) Using a bur, distal bone may be removed and the tooth may be dislodged distally. Then the tooth is removed.

(b) The distal half of the crown may be sectioned by slicing from the buccal groove to a point just below the cervical line on the distal side of the tooth. This slice is removed, and then the remainder of the tooth is delivered using a straight elevator placed on the mesial aspect.

(c) The point of application of the elevator is changed from mesial to the buccal side and a firm upward force is exerted. A purchase point can be created with a bur and the toot can be delivered using a Cryer's elevator.

Sometimes, the mesioangular tooth may be entrapped beneath the distal convexity of the crown of the second molar. In such cases, the tooth may be divided at the cervical region to separate the crown, which is then removed by applying force beneath its inferior surface. The roots may then be delivered by engaging at the bifurcation.

If the tooth roots are in close contact with the mandibular canal, applying levering force can force the root apex downward which may damage the neurovascular bundle. Crown sectioning must be preferred in such cases, which will allow the roots to be delivered upward away from the canal. This would prevent damage to the canal.

The horizontally impacted (Fig. 14.28a, b, c, d) (Video 14.2) tooth may need more bone to be removed as compared to mesioangular impaction. A deeply impacted tooth tends to engage either the crown or root of the second molar. This makes its removal difficult. Adequate bone is removed superiorly to expose the entire crown width, as well as the upper third of the root. The point of application of elevator is pro- 


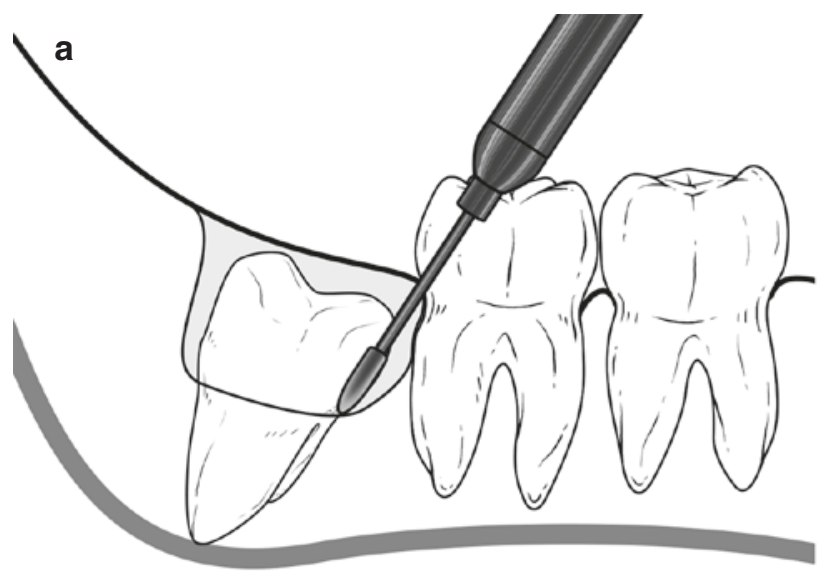

b
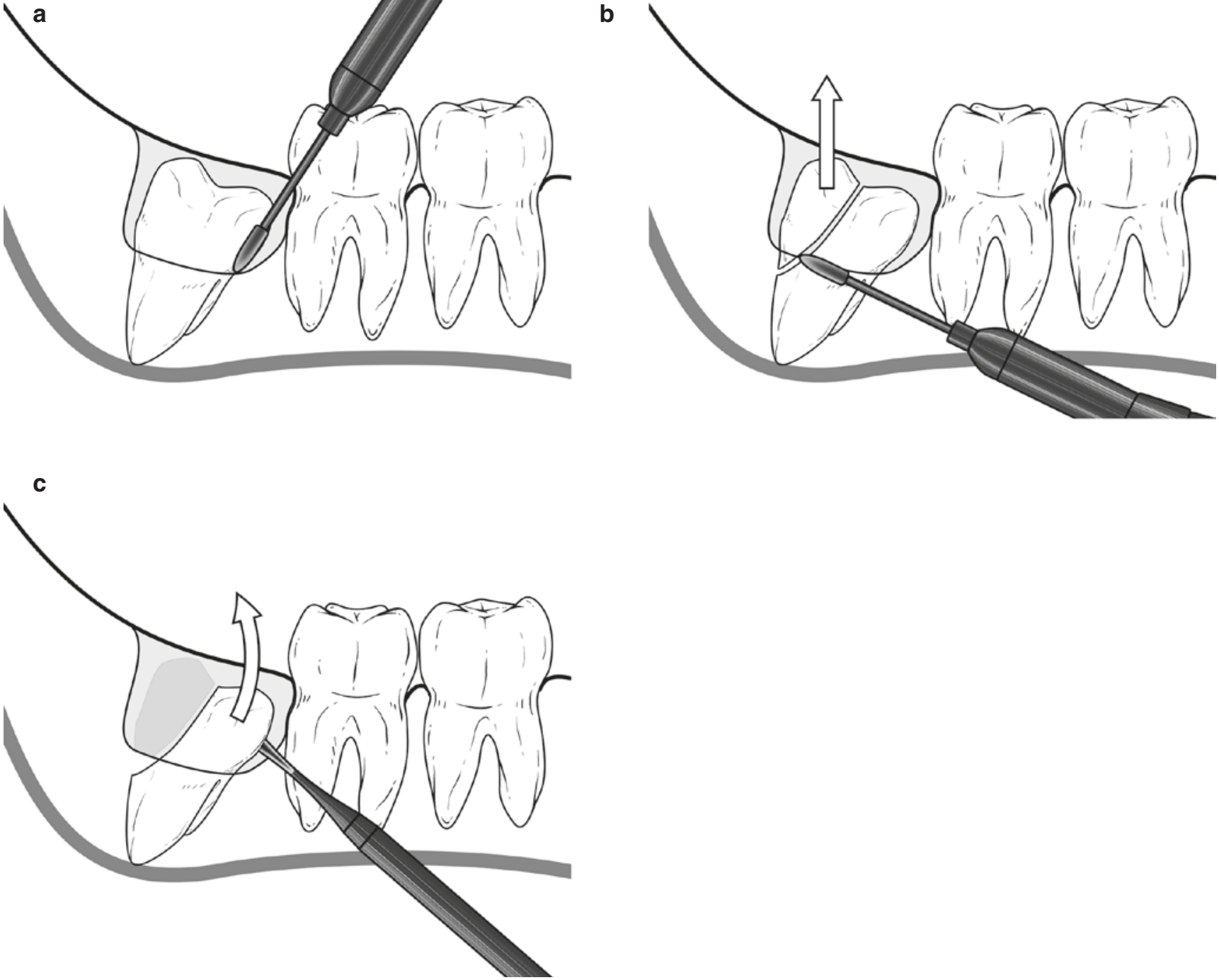

CAssociation of Oral and Maxillofacial Surgeons of India

Fig. 14.27 (a, b, c) (see text for details) Steps in the surgical removal of mesioangular impaction. (a) Bone removed up to cemento enamel junction using bur, (b) Sectioning of tooth, (c) Tooth delivery using elevator

cured below the mesiobuccal aspect of the impacted crown. The tooth is then sectioned at the cervical region and the crown is removed from the socket. The root is then brought forward into the vacant space previously occupied by the crown and it is then removed either in a single piece or after sectioning.

In cases where the impacted tooth is not locked beneath the distal convexity of the crown of the second molar and when an adequate amount of distal bone has been removed, it is possible to turn the tooth into a vertical position by application of force in the mesial aspect. This is similar to the procedure already described for the removal of mesioangular impactions. Use of further force with the elevator will expel the tooth out of the socket or force can be applied on the buccal side to remove the tooth.

Another method of removing horizontal impactions is to split the tooth horizontally into two by sectioning via the buccal groove into separate mesial and distal roots (technique shown in Fig. 14.29). The distal root with the attached crown part is elevated out first followed by the deeply lying mesial root and part of the crown. If there is difficulty in elevating the deeply placed mesial root segment, it can be again sectioned into two at the cervical region and the crown and the root parts may be removed separately.

The vertical impaction (Fig. 14.29a, b, c) is one of the more difficult ones to remove, especially if it is impacted very deeply. The procedure for bone removal and the sectioning is similar to that of a mesioangular impaction. Here also the bone is removed first from the occlusal, buccal, and distal aspect. The distal half of the crown is then sectioned and removed, and the tooth is elevated by applying a small straight elevator at the mesial aspect of the cervical line. Alternatively, similar to mesioangular impactions, a pur- 


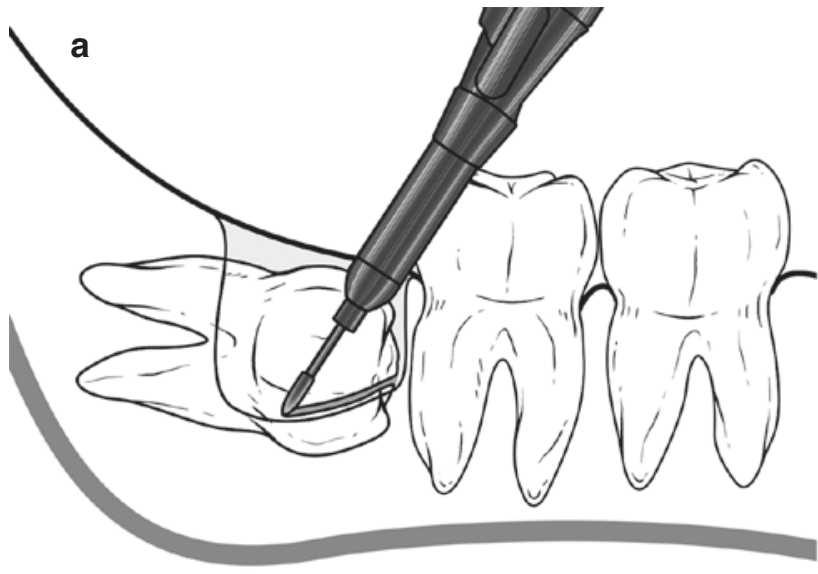

C

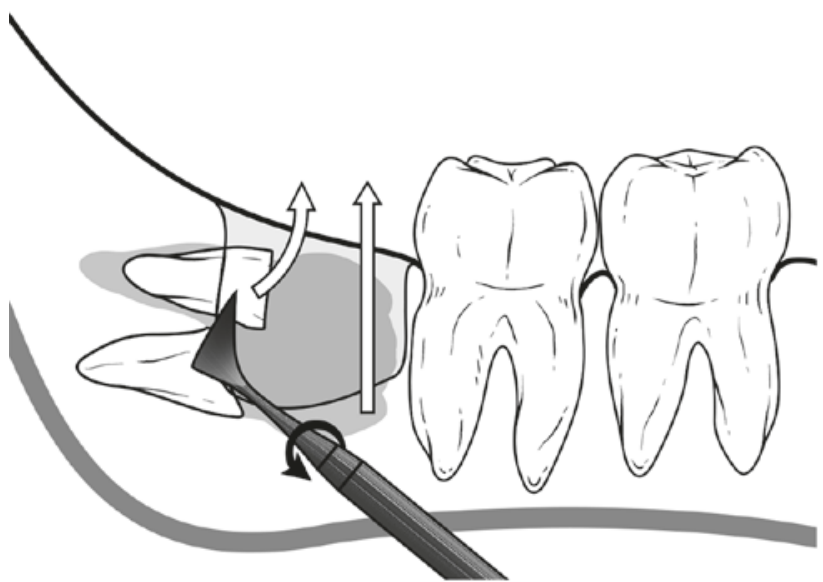

CAssociation of Oral and Maxillofacial Surgeons of India

Fig. 14.28 (a, b, c, d) Steps in the surgical removal of a horizontally impacted mandibular third molar. (a) Bone removal to expose the width of the crown and the upper third of the root, (b) Crown may be sectioned into two as shown in the figure and elevated separately. Another technique is to divide the tooth at cemento enamel junction and elevate the crown as a single piece, (c) After removal of the crown, the distal

chase point can be made on the buccal side of the tooth, and a Cryer's elevator may be used to deliver the tooth.

The distoangular impaction (Fig. 14.30a, b, c) is considered to be the most difficult tooth to remove. The goal of the technique for removal of these teeth is to create an adequate buccal and distal trough (guttering) around the crown of the tooth to a depth below the cervical line. This will permit to make a point of application of elevator on the buccal aspect of the tooth. Then, using the buccal cortical plate as the fulcrum, force is applied to elevate the tooth out of the socket upward and distally. If some movement is obtained, the distal portion of the crown or the complete crown can be sectioned in a horizontal fashion from the roots and removed. It is preferable in this case to section the tooth segments further as needed rather than to remove more bone. It would be wise to remember the adage b

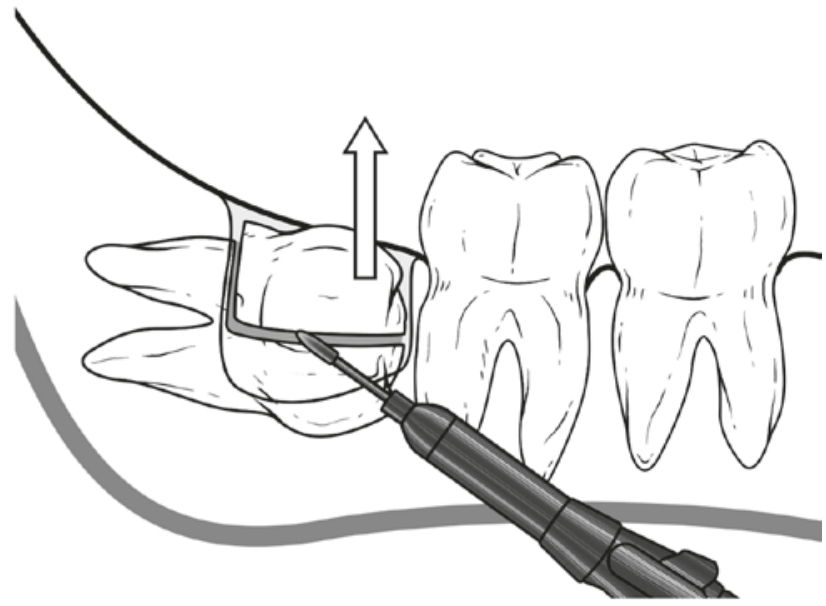

d

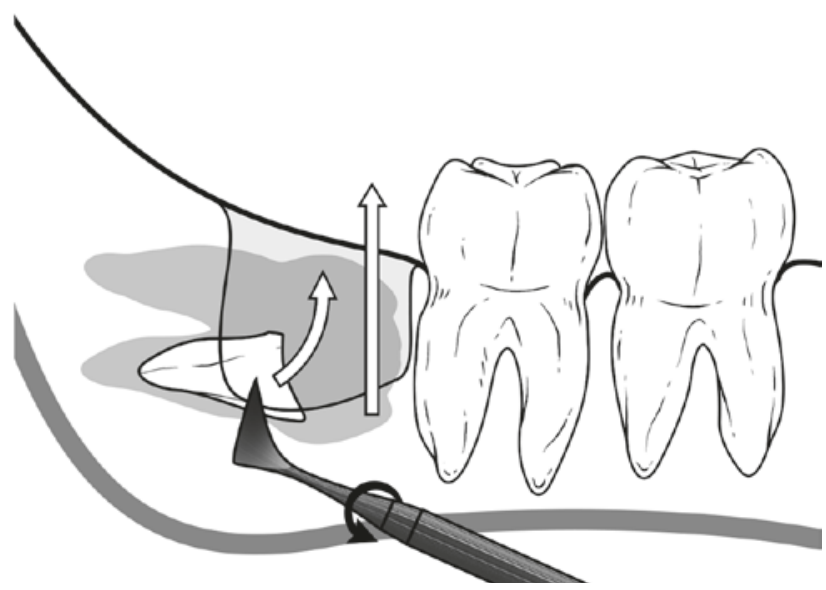

root sectioned at the furcation is brought forward into the space occupied by the crown, (d) Removal of the mesial root. (the technique shown in Fig. 14.29 can also be used for horizontal imapctions where the tooth is sectioned via the bifurcation and distal crown and root is elevated out first, followed by the mesial crown and root)

that "Tooth belongs to the surgeon and Bone belongs to the patient". This will ensure preservation of the structural integrity of the mandible. The roots are then delivered together or sectioned and delivered independently with a Cryer's elevator.

In cases where tooth sectioning is required, the distal root should be elevated first followed by the mesial root.

\subsubsection{Debridement}

After tooth delivery, all bone debris and tissue must be removed from the socket. This is best accomplished by irrigation with saline and mechanically debriding the socket and the area under the flap with a cruet. A bone file or a large bur is used to smooth any rough and sharp edges of the 


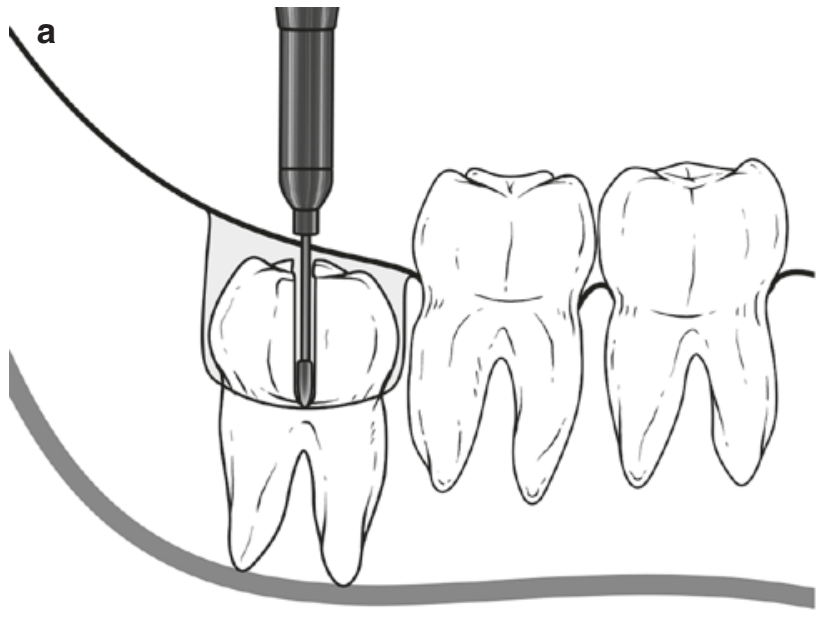

C

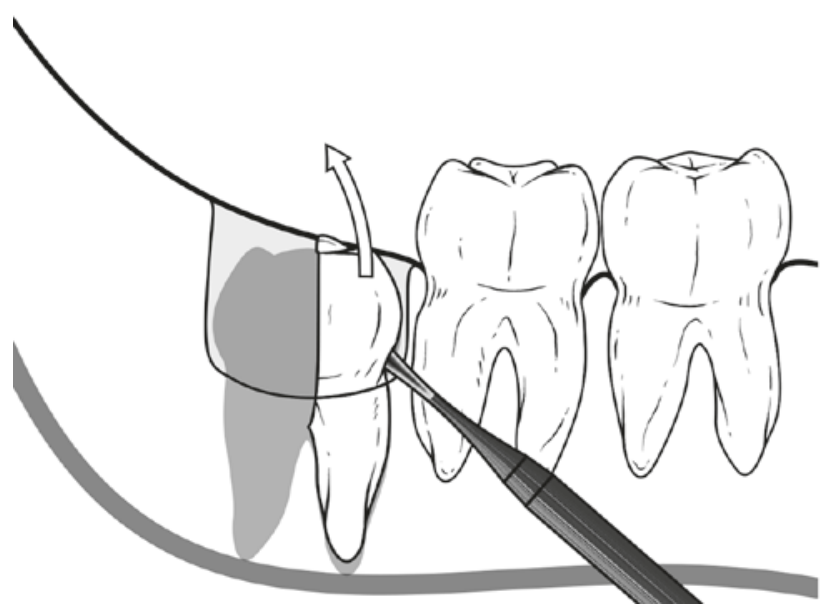

CAssociation of Oral and Maxillofacial Surgeons of India

Fig. $14.29(\mathbf{a}, \mathbf{b}, \mathbf{c})$ Steps in the surgical removal of a vertically impacted mandibular third molar. (a) Bone removal to expose the width of the crown, (b) Distal half of the crown sectioned up to the furcation

bone. Any remaining dental follicle must be removed using a mosquito hemostat, to prevent cyst formation. An artery forceps may be used to remove fractured interdental septum or large pieces of bone. The socket and the wound margins (including under surface of mucoperiosteum) is irrigated with saline or sterile water to remove bone and tooth debris.

\subsubsection{Wound Closure}

Before attempting closure, bleeding from the socket is completely arrested. Further bleeding from the socket can be controlled using bone wax, Surgicel, or Gelfoam. If there is bleeding from the socket underneath a tight suture, blood will accumulate in surrounding tissue spaces leading to buccal or lingual hematoma or ecchymosis. The flap is then b

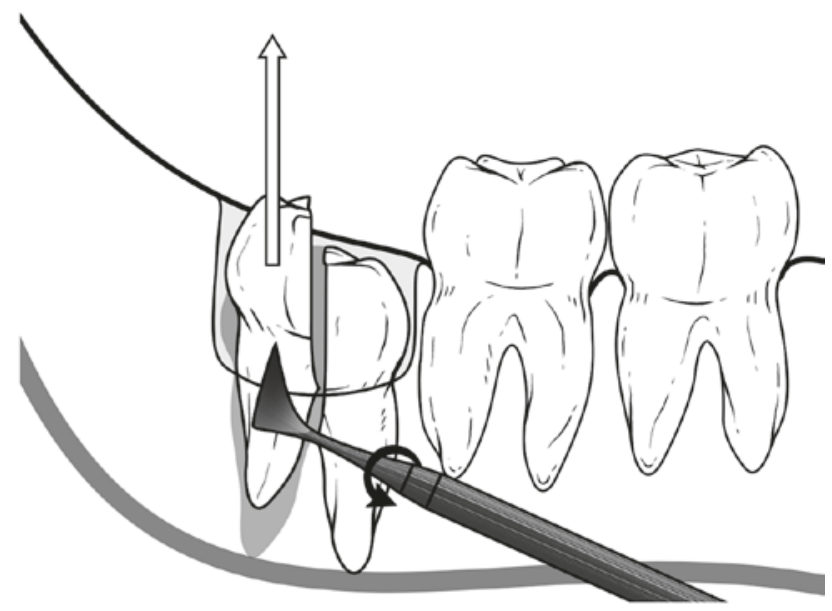

and it is removed along with the root, (c) Mesial half of the tooth is elevated by mesial application of force at the cervical line

replaced to its original position and the initial suture placed just distal to the second molar. This suture reduces the possibility of the development of periodontal pocket distal to the second molar. The needle is passed from the buccal to the lingual side. Additional sutures are then placed as necessary. The sutures should be just tight enough to hold the flap. Over tightening should be avoided. The vertical component of the incision is left unsutured since it will act as a wound toilet.

Following the procedure, oral and written postoperative instructions given to patient and bystander ensure better patient compliance.

The influence of lower third molar impactions on the periodontal health of the adjacent second molar and the influence of third molar removal on the periodontal attachment of the second molar is a very contentious topic and multiple studies have been done in this regard [32]. 


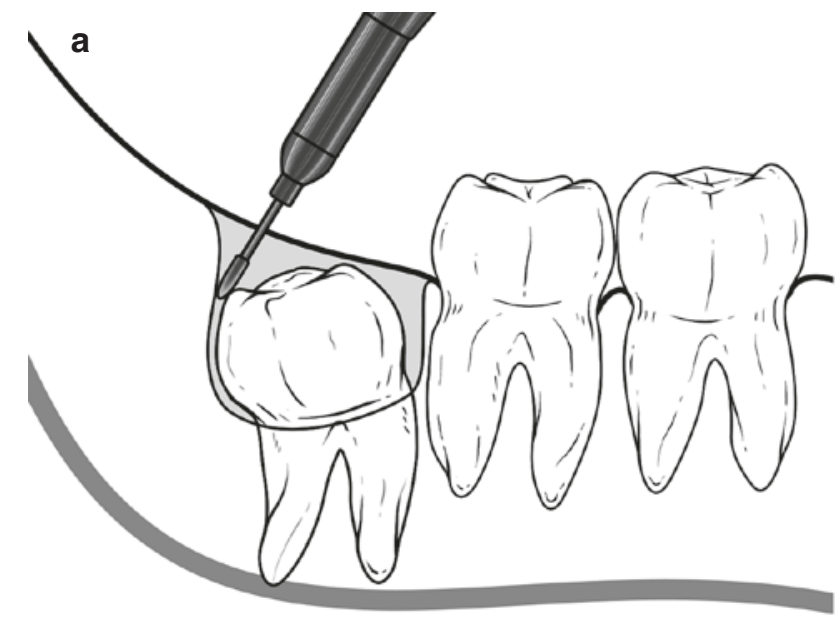

C

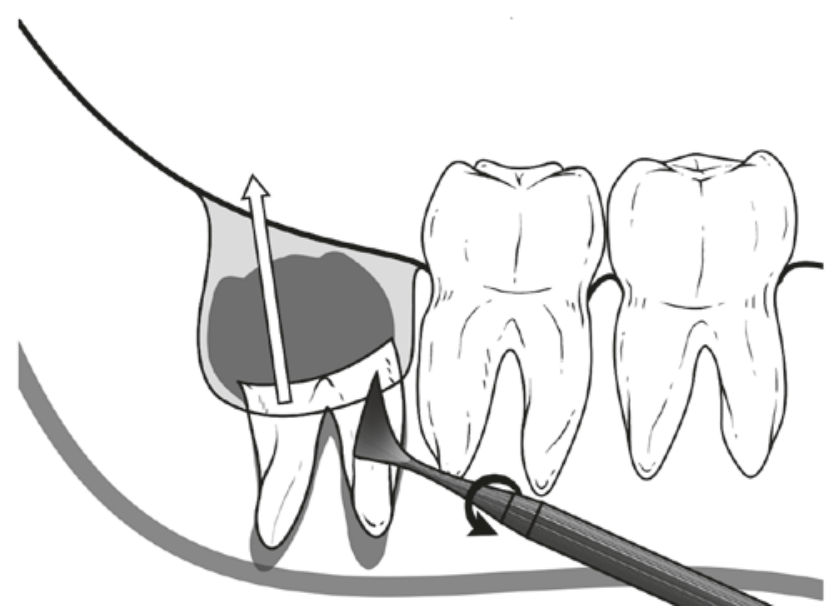

CAssociation of Oral and Maxillofacial Surgeons of India

Fig. $14.30(\mathbf{a}, \mathbf{b}, \mathbf{c})$ Steps in the surgical removal of a distoangularly impacted mandibular third molar. (a) Bone removed to expose the full crown of the tooth to a depth below the cervical line, (b) Crown sectioned in a horizontal fashion from the roots and removed (some sur-

\subsubsection{Other Methods for Removal/Partial Removal of Impacted Lower Third Molar}

In addition to the standard surgical technique described above, there may be occasions where other methods of surgical removal also have to be considered. This is because no technique is suited to every case and it will be ideal to learn the different methods and choose the suitable one depending upon the case. Readers are advised to refer the concerned publications to get more details. b

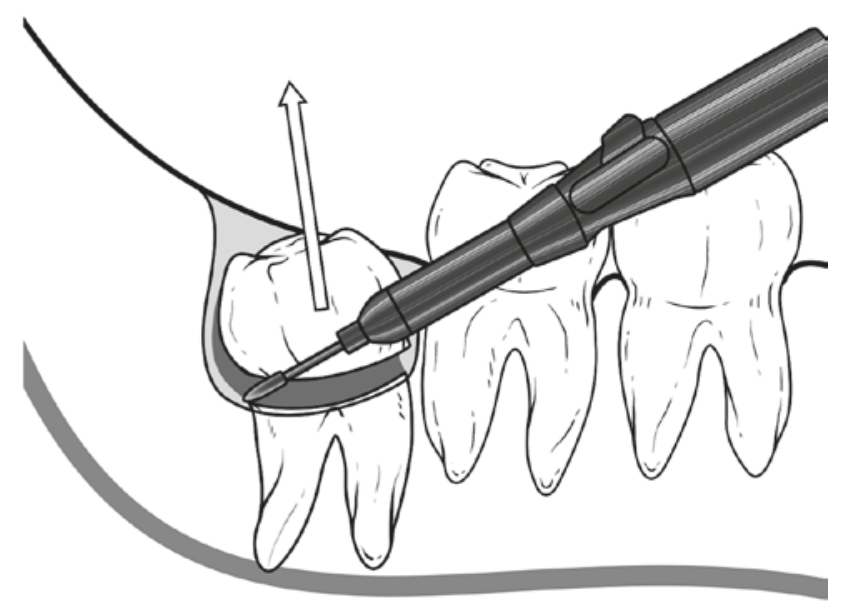

geons prefer to remove only the distal portion of the crown of third molar, so that a point of elevation is available distal to the second molar tooth), (c) Roots are then delivered together or sectioned and delivered independently with a Cryer's elevator
Some of the other methods seen in the literature are

1. Removal via sagittal split osteotomy [33].

2. Buccal corticotomy technique [34].

3. Lingual split bone technique $[35,36]$.

4. Lateral trephination technique $[37,38]$.

5. Partial odontectomy/Coronectomy $[15,16,39]$.

6. Removal of the tooth after orthodontic extrusion $[40,41]$. 


\subsection{Impacted Maxillary Third Molar (Video 14.3)}

Surgical management of upper third molars in general is less complicated compared to lower third molars. They cause less discomfort, are more likely to erupt and are simpler to remove unless unerupted and encased in bone. Removal of upper third molars results in far less postoperative morbidity.

The commonest type of impaction in maxillary third molar is vertical [42].

Classification of impacted maxillary third molars-The system of classification of impacted upper wisdom tooth is basically the same as that for mandibular third molar. However, there are some additional parameters to be considered which will aid in preoperative assessment of the case and guide in planning the surgery for a successful outcome.

\section{State of eruption.}

(a) Fully erupted.

(b) Partially erupted.

(c) Unerupted:

- within the bone

- immediately beneath the soft tissues

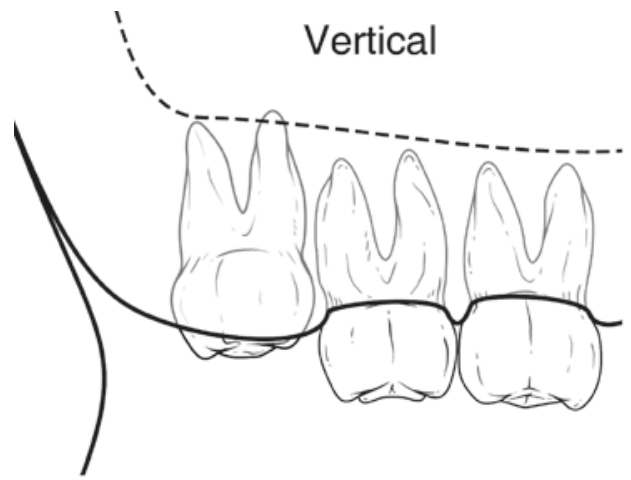

2. Angulation of the tooth (Fig. 14.31).

(a) Vertical.

(b) Mesioangular.

(c) Distoangular.

(d) Laterally displaced with the crown facing the cheek, horizontal, inverted, and transverse positions.

(e) Aberrant position sometimes associated with pathological condition such as cyst.

3. Pell and Gregory classification-This is based on the relative depth of the impacted maxillary third molar, (Fig. 14.32). Position A-Occlusal surface of the third molar is at the same level as that of the second molar.

Position B-Occlusal surface of the third molar is located between the occlusal plane and cervical line of the second molar.

Position C-Occlusal surface of the third molar is at or above the cervical line of the second molar.

4. Relationship of impacted maxillary third molar to the maxillary sinus.

(a) Sinus approximation (SA) - No bone or a thin partition of bone between the impacted maxillary third molar and maxillary sinus.

(b) No sinus approximation (NSA) $-2 \mathrm{~mm}$ or more bone between the impacted maxillary third molar and maxillary sinus.

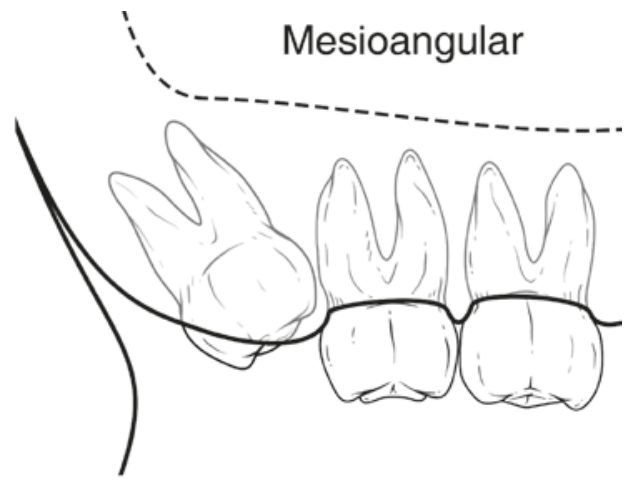

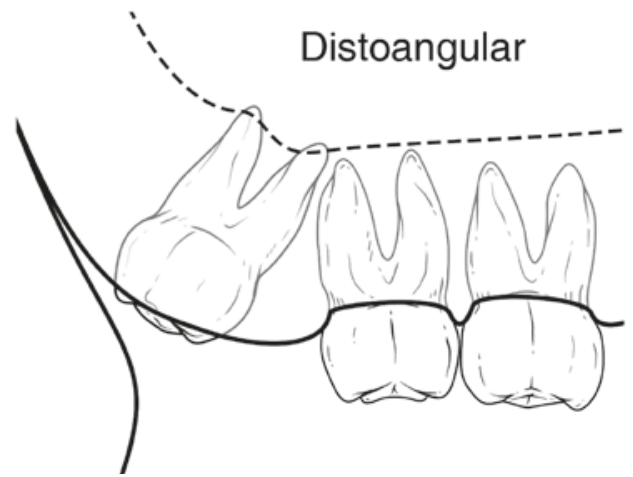

CAssociation of Oral and Maxillofacial Surgeons of India

Fig. 14.31 Classification of impacted maxillary third molar based on angulation 

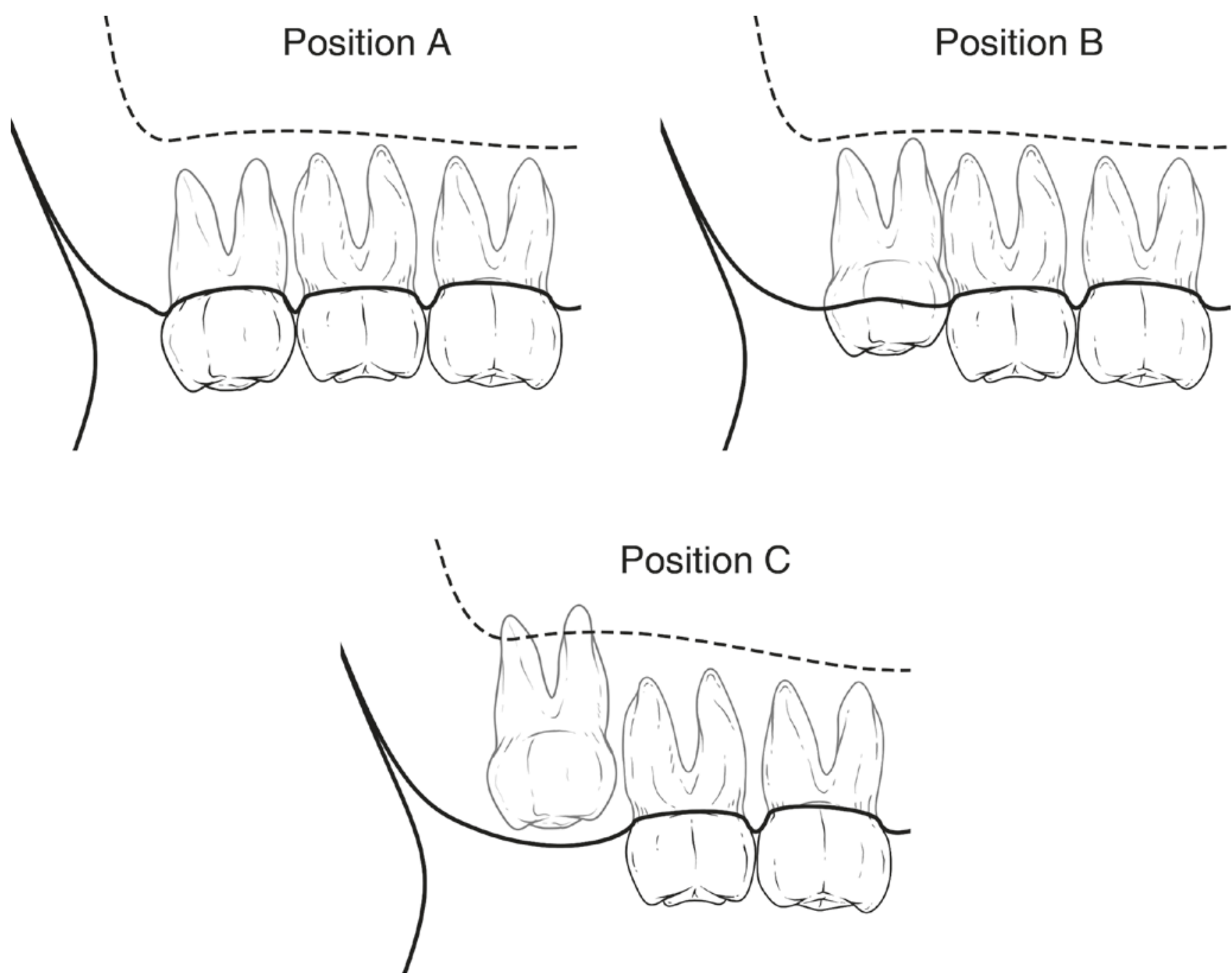

CAssociation of Oral and Maxillofacial Surgeons of India

Fig. 14.32 Pell and Gregory classification based on relative depth of impacted maxillary third molar

\section{Nature of roots.}

(a) Fused (conical).

(b) Multiple-Favorable/Unfavorable.

\subsubsection{Radiographic Examination}

The following are the useful radiographs:

1. Periapical X-ray.

2. Orthopantomogram (OPG).

3. Occlusal X-ray.

4. True lateral view—occasionally helpful.

5. Paranasal sinus view: useful to view pathologies associated with the tooth.

6. CT scan-more useful for suspected pathologies in relation to the impacted tooth.

\subsubsection{Indications for the Removal of Maxillary Third Molar}

1. Unrestorable dental caries.

2. Recurrent pericoronitis.

3. A tooth that has erupted in a buccoverted or distal direction, which cause cheek bite, or abnormal bite patterns.

4. Tooth involved in pathological process such as cyst.

5. Overerupted and nonfunctional upper third molar.

6. Interference with the placement of prosthesis.

\subsubsection{Adjacent Anatomical Factors to be Considered: (Fig. 14.33a-d)}

- Proximity to maxillary sinus (Fig. 14.34).

- Proximity to maxillary tuberosity. 

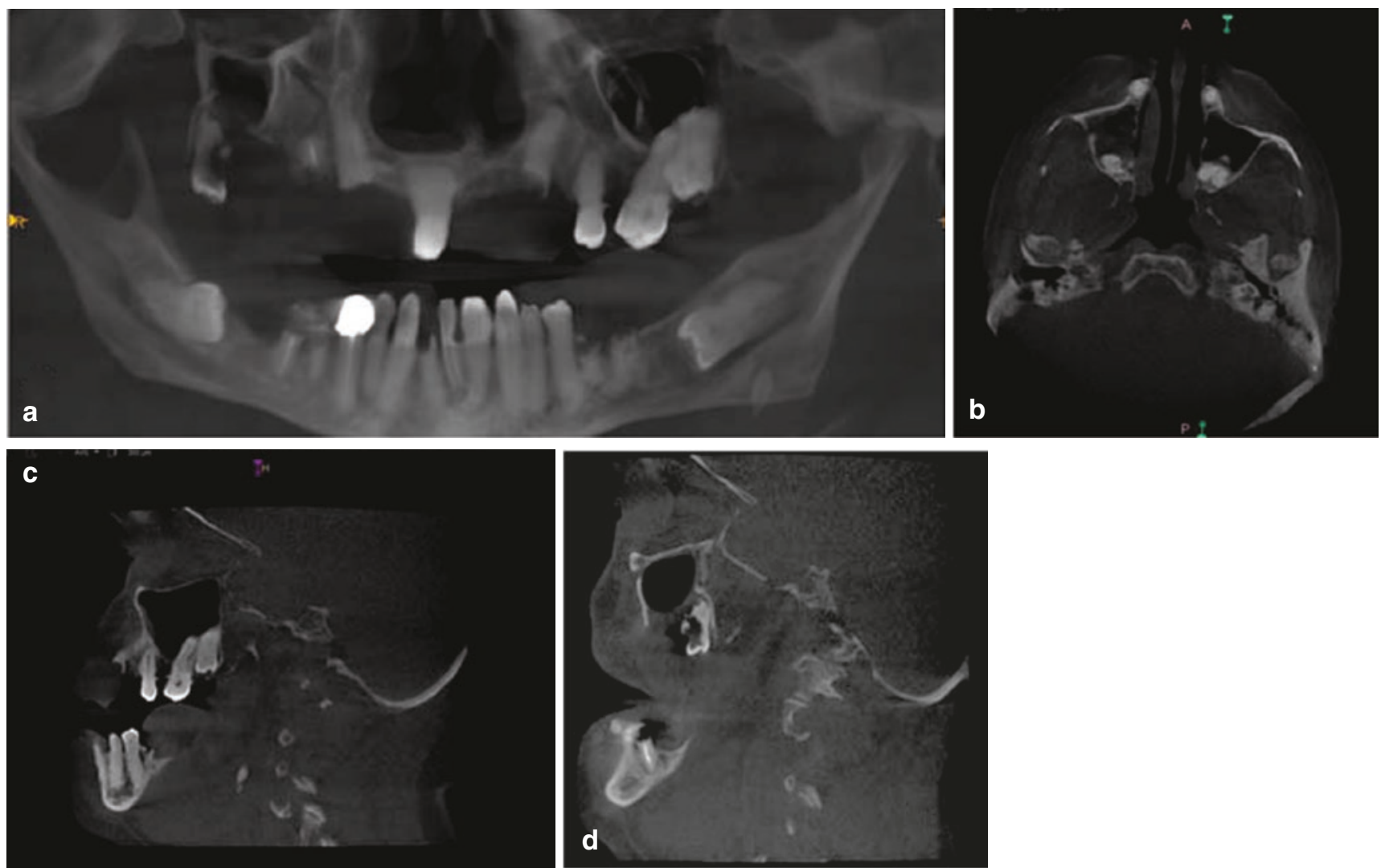

CAssociation of Oral and Maxillofacial Surgeons of India

Fig. 14.33 (a-d) Impacted third molars in a 75-year-old male. (a) OPG showing impacted 28,38 , and 48 with multiple root stumps and dental caries for 18. (b) Axial CBCT showing close relationship of 18 and 28 to maxillary sinus, (c) Sagittal view showing sinus approximation of roots of 27 and 28, (d) Sagittal view showing close relationship of 18 to sinus floor
- Buccal pad of fat.

- Pterygopalatine fossa.

- Infratemporal fossa.

\subsubsection{Surgical Removal of Impacted Maxillary Third Molar (Figs. 14.35a-d and 14.36a, b)}

The main difficulty here is that the coronoid process may block access to this region, which may be overcome by limiting the amount of mouth opening.

The procedure for maxillary third molar surgical removal is almost the same as that of the mandibular third molar.

\subsubsection{Incision}

It starts from the mesial aspect of the first molar and extends distally beyond the distobuccal aspect of the second molar and is then continued into the tuberosity. In case of a deeply impacted tooth if greater access is required, a triangular flap may be raised by placing a release incision mesial to the second molar.
The mucoperiosteum is then reflected using a Howarth's periosteal elevator, which may also be used to retract the flap.

\subsubsection{Removal of Overlying Bone}

Bone removal is generally limited to the occlusal and the buccal aspect of the tooth down to the cervical line to expose the entire crown (Figs. 14.35b and 14.36a). This is done using bur. To create space for the elevator to be inserted, more bone may be removed from the mesial part of the tooth, at a point above the maximum bulge of the crown.

Unlike mandibular third molars, maxillary third molars rarely need sectioning, as maxillary bone expands easily, being thin and elastic. Instances where the bone is thicker, sclerotic and less elastic as in old patients, tooth removal is enabled by bone removal rather than tooth sectioning. Chisel is contraindicated to section the tooth due to the danger of displacement of the tooth into the maxillary antrum.

Maxillary third molar teeth must not be sectioned unless absolutely necessary, as displacement of small fragments into the sinus or infratemporal fossa may occur. 


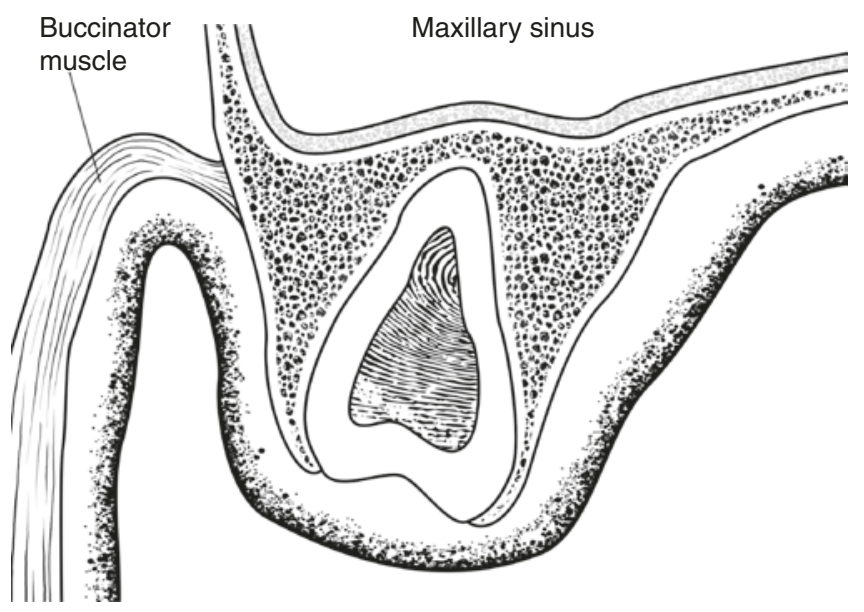

CAssociation of Oral and Maxillofacial Surgeons of India

Fig. 14.34 Schematic diagram showing the relationship of impacted maxillary third molar to the floor of the maxillary sinus
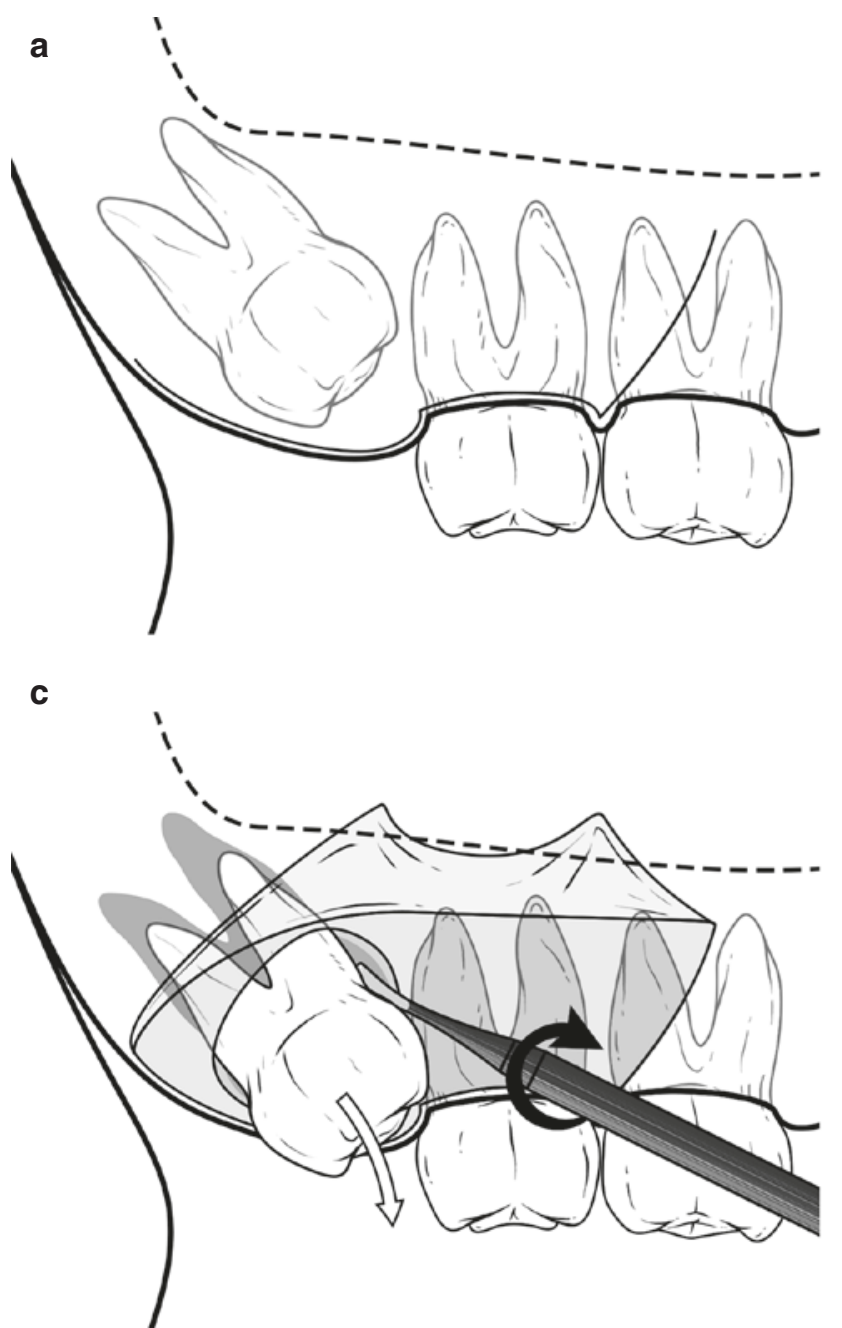

CAssociation of Oral and Maxillofacial Surgeons of India

Fig. 14.35 (a-d) Steps in the surgical removal of a mesioangularly impacted maxillary third molar. (a) Incision to raise a triangular flap, (b) Mucoperiosteal flap reflected, (c) Overlying bone removed from

\subsubsection{Delivery of the Tooth}

This is achieved using small straight elevators or angled elevators with force exerted in the distobuccal direction. If angled elevators are used, access may be easier. Angled elevators which can be used for this purpose are the Warwick James, Cryer, Pott's, and the Apex elevator. During surgical removal, placement of Laster retractor will help in better access and vision and may also prevent accidental displacement of the maxillary third molar into tissue space beyond the tuberosity.

During tooth elevation, one must remember the following points:-

1. Due to the proximity of the maxillary sinus and the infratemporal fossa, pressure should not be exerted in the superior direction during bone removal and delivery of the tooth.

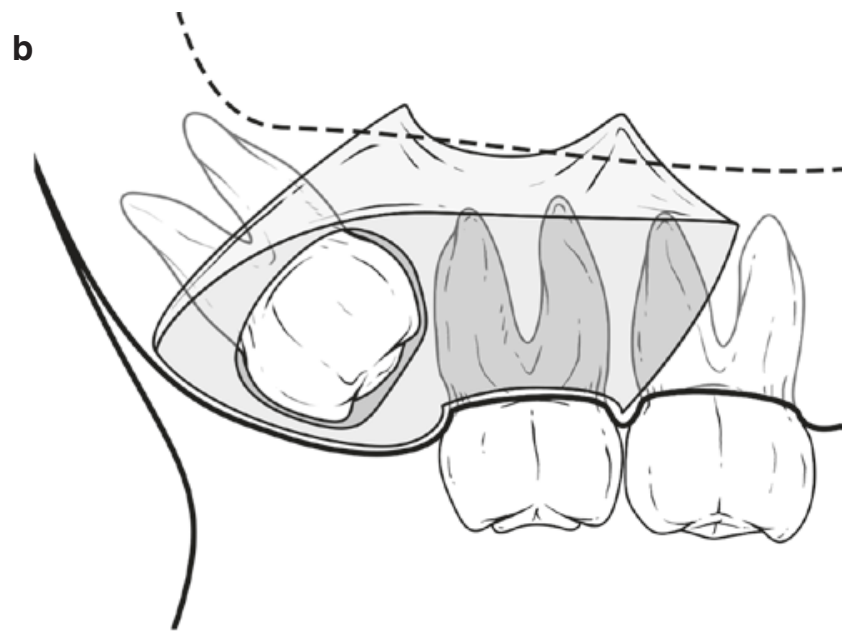

d

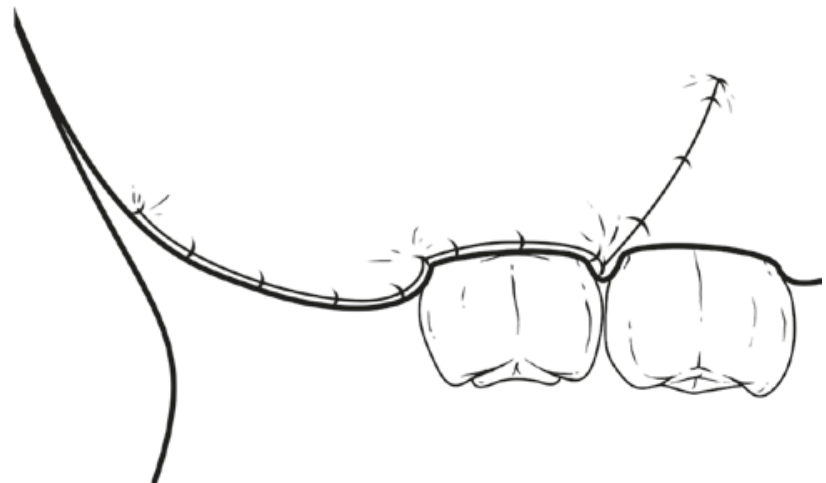

occlusal and buccal aspect up to the cervical line and elevation of tooth, (d) Suturing completed 

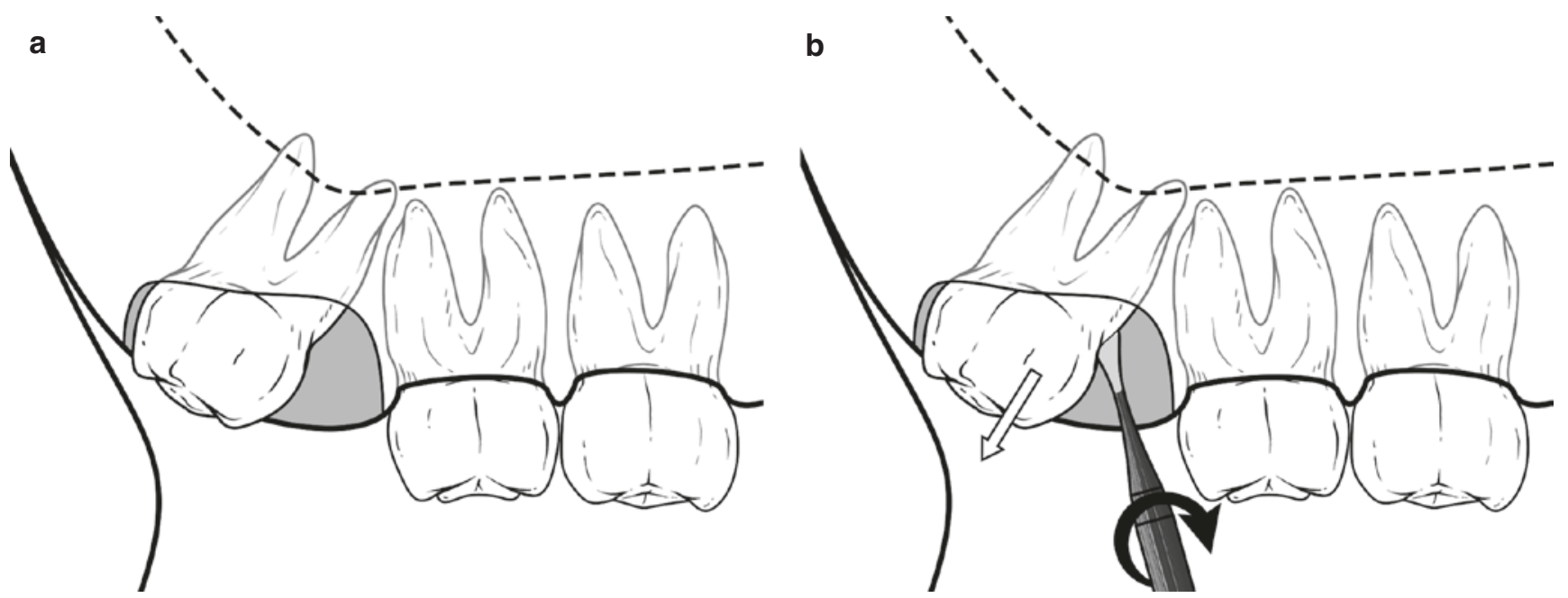

CAssociation of Oral and Maxillofacial Surgeons of India

Fig. 14.36 (a) Bone removal achieved on the occlusal and the buccal aspect of tooth down to the cervical line to expose the entire crown, in a disto angular maxillary third molar impaction (b) Delivery of the tooth using an elevator

2. Sufficient space is created between the height of contour of the crown (i.e. above the maximum bulge of the tooth) and surrounding bone so that the tip of the elevator can be placed above the height of contour of the tooth. Then pressure is exerted in a distobuccal direction.

3. Moderate pressure is then exerted distally, buccally and occlusally (i.e. downward and outward) with the fore finger placed posterior to maxillary tuberosity to detect tuberosity fracture if it occurs.

4. In case an accidental opening into the sinus is suspected, every effort should be made to ensure proper closure and the patient is instructed appropriately to prevent the development of an oro-antral fistula.

\subsubsection{Complications That Occur During Surgical Removal of Impacted Maxillary Third Molar}

1. Tooth displacement into maxillary sinus-This usually occurs in cases of partially erupted maxillary third molars, which have conical roots and are located close to the floor of the sinus. The risk increases if the root apex is in contact with the floor of the sinus, and the initial position of the tooth is high.

If this complication occurs, the tooth may have to be removed from the sinus in order to avoid infection. Initially, a suction tip may be placed at the sinus opening to retrieve the tooth. Alternately, saline irrigation into the sinus may be followed by applying the suction tip. If these methods do not work, it is best to stop attempts and place the patient on antibiotics and nasal decongestants. The tooth may be retrieved later through Caldwell-Luc approach, and the oro-antral fistula may then be closed. The tooth may also be removed using endoscopic sinus surgery [43, 44]. Detailed sequential approach of dealing with root/ tooth displaced into the sinus is mentioned in the Chap. 24 on Oro antral communication and fistula in this text book.

2. Dislodgement into soft tissues-The upper third molar can be inadvertently displaced into the buccal soft tissues [45] or into the infratemporal fossa [46]. Usually, this happens: (a) when the flap raised buccally is not adequate for access, (b) there is insufficient visibility during the procedure, (c) improper extraction technique, (d) distolingual angulation of tooth, and (e) the crown of the third molar is at a level above the root apices of the adjacent molar tooth.

Dimitrakopoulos et al. [46] have discussed the various methods to remove a maxillary third molar that has been displaced into the infratemporal fossa.

3. Damage to adjacent second molar.

4. Fracture of maxillary tuberosity.

5. Oro-antral communication/fistula.

\subsection{Complications of Impaction Surgery}

Complications of removal of impacted tooth can happen during the procedure and late after the procedure. Mild post operative pain, swelling and trismus can be expected 
inmost case and these three can be considered as a sequel ae of surgical removal of impacted wisdom teeth:-
(a) The actual surgical procedure.
(b) Late after surgery.

\section{A. Complications that can occur during the Surgical Procedure:}

These can happen at various steps in the procedure, including:

1. Placement of incision.

2. Removal of bone.

3. Sectioning of the tooth.

4. Tooth elevation and delivery.

1. Complications during incision:

(a) Bleeding from retromolar vessels.

(b) Bleeding from facial vessels.

(c) Damage to lingual nerve.

2. Complications during bone removal:

(a) Use of bur.

- Accidental burns.

- Laceration of soft tissues.

- Injury to inferior alveolar neurovascular bundle.

- Injury to adjacent tooth.

- Injury to lingual nerve.

- Necrosis of bone.

- Emphysema.

(b) Use of chisel

- Splintering of bone.

- Fracture of mandible.

- Displacement of tooth into lingual pouch.

- Injury to lingual nerve.

- Injury to the soft tissues or second molar.

3. Complications during sectioning of tooth.

During bur usage

- Sectioning along an incorrect line.

- Injury to mandibular canal.

- Breakage of bur.

4. Complications during elevation of tooth:

- Fracture of impacted tooth/root.

- Injury to second molar.

- Fracture of mandible.

- Displacement of the entire tooth or crown alone into the lingual pouch or lateral pharyngeal space.

\section{B. Postsurgical Sequelae and Complications}

1. Pain

2. Edema

3. Trismus

4. Haemorrhage

5. Infection

6. Alveolar osteitis (Dry socket)

7. Nerve Injury:

(a) Lingual nerve injury

(b) Inferior alveolar nerve injury

8. Surgical Emphysema

9. Hematoma

10. Pain during swallowing

11. Pyrexia

12. Osteomyelitis

13. Temporomandibular joint (TMJ) complications

14. Fracture of instruments

15. Periodontal pocket formation distal to second molar

16. Aspiration/Swallowing of tooth

The management of impacted third molars involves several considerations, and several controversies also exist in this area. Some of these are as follows:

- Whether to remove Asymptomatic/Disease-free tooth [4-6].

- Age of removal [47].

- Flap designs [20-22].

- Technique of removal: bur/laser [31]/peizo surgery $[29,30]$.

- Primary closure of socket/secondary closure of socket [48].

- Drains in socket/No drains in socket [49].

- Whether to graft the Socket with PRP [50]/PRF [51-53].

- Reconstruction of distal periodontal defect of the second molar [54].

At present, the various evidence-based guidelines available should help the clinician in taking informed decisions regarding third molar impactions [55-57]. Efforts have been made to reach a consensus in various areas, which itself shows conflicting propositions, and only time will prove the best methods which can be used in the management of third molar impactions. 


\section{References}

1. Archer WH. Oral and maxillofacial surgery, vol. I. 5th ed. Philadelphia: W.B. Saunders; 1975.

2. Lytle JJ. Indications and contraindications for removal of impacted tooth. Dent Clin of North Amer. 1979;23(3):333-46.

3. Andreasen J.O, Petersen J.K, Laskin D.M. Textbook and color atlas of tooth impactions, 1, Copenhagen, Munksgaard 1997.

4. Dodson TB. The management of the asymptomatic, disease-free wisdom tooth: removal versus retention. Atlas Oral Maxillofac Surg Clin North Am. 2012 Sep;20(2):169-76.

5. Rafetto LK. Removal of asymptomatic third molars: a supporting view. J Oral Maxillofac Surg. 2006 Dec;64(12):1811-5.

6. Hill CM. Removal of asymptomatic third molars: an opposing view. J Oral Maxillofac Surg. 2006 Dec;64(12):1816-20.

7. Berger A. As cited by Archer, W. H., Oral and maxillofacial surgery, vol. I, 5th ed. Philadelphia: W.B. Saunders; 1975.

8. Pogrel MA, Renaut A, Schmidt B, et al. The relationship of the lingual nerve to the mandibular third molar region: an anatomic study. J Oral Maxillofac Surg. 1995;53:1178-81.

9. Behnia H, Kheradvar A, Shahrokhi M. An anatomic study of the lingual nerve in the third molar region. J Oral Maxillofac Surg. 2000;58:649-51.

10. Winter GB. Principles of exodontia as applied to the impacted third molar. St Louis: American Medical Books; 1926.

11. Pell GJ, Gregory BT. Impacted mandibular third molars: classification and modified techniques for removal. Dent Digest. 1933;39:330-8.

12. Suvy Manuel, Surej Kumar LK, Mathew P Varghese. A comprehensive proforma for evaluation of mandibular third molar impactions. J Maxillofac Oral Surg. 2014 Dec; 13(4):378-385.

13. Howe G, Poynton HG. Prevention of damage to the inferior alveolar nerve during the evaluation of mandibular third molars. Br Dent J. 1960;109:355-63.

14. Rood JP, Shehab BA. The radiological prediction of inferior alveolar nerve injury during third molar surgery. Br J Oral Maxillofac Surg. 1990 Feb;28(1):20-5.

15. Pogrel MA, Lee JS, Muff DF. Coronectomy: a technique to protect the inferior alveolar nerve. J Oral Maxillofac Surg. 2004;62:1447.

16. Renton T, Hankins M, Sproate C, McGurk M. A randomised controlled clinical trial to compare the incidence of injury to the inferior alveolar nerve as a result of coronectomy and removal of mandibular third molars. Br J Oral Maxillofac Surg. 2005;43:7.

17. Tantanapornkul W, Okouchi K, Fujiwara Y, et al. A comparative study of cone-beam computed tomography and conventional panoramic radiography in assessing the topographic relationship between the mandibular canal and impacted third molars. Oral Surg Oral Med Oral Pathol Oral Radiol Endod. 2007;103:253.

18. Ohman A, Kivijarvi K, Blomback U, Flygare L. Pre-operative radiographic evaluation of lower third molars with computed tomography. Dentomaxillofac Radiol. 2006;35:30.

19. Danforth RA, Peck J, Hall P. Cone beam volume tomography: an imaging option for diagnosis of complex mandibular third molar anatomical relationships. JCDA. 2003;31(11):847-52.

20. Gargallo-Albiol J, Buenechea-Imaz R, Gay-Escoda C. Lingual nerve protection during surgical removal of lower third molars. J Oral Maxillofac Surg. 2000;29:268.

21. Pichler JW, Beirne OR. Lingual flap retraction and prevention of lingual nerve damage associated with third molar surgery: a systematic review of the literature. Oral Surg Oral Med Oral Pathol Oral Radiol Endod. 2001;91:395.

22. Pogrel MA, K.E. Goldman: lingual flap retraction for third molar removal. J Oral Maxillofac Surg. 2004;62:1125.
23. Gulicher D, Gerlach KL. Sensory impairment of the lingual and inferior alveolar nerves following removal of impacted mandibular third molars. Int J Oral Maxillofac Surg. 2001;30:306.

24. Valmaseda-Castellon E, Berini-Aytes L, Gay-Escoda C. Inferior alveolar nerve damage after lower third molar surgical extraction: a prospective study of 1117 surgical extractions. Oral Surg Oral Med Oral Pathol Oral Radiol Endod. 2001;92:377.

25. Pederson GW. Oral surgery. Philadelphia: WB Saunders; 1988. (Cited in: Koerner KR. The removal of impacted third molarsprinciples and procedures. Dent Clin North Am. 1994; 38:255-78)

26. Yuasa H, Kawai T, Sugiura M. Classification of surgical difficulty in extracting impacted third molars. Br J Oral Maxillofac Surg. 2002 Feb;40(1):26-31.

27. Erdogan O, Tatlı U, Ustün Y, Damlar I. Influence of two different flap designs on the sequelae of mandibular third molar surgery. Oral Maxillofac Surg. 2011 Sep;15(3):147-52.

28. Goldsmith SM, De Silva RK, Tong DC, Love RM. Influence of a pedicle flap design on acute postoperative sequelae after lower third molar removal. Int J Oral Maxillofac Surg. 2012 Mar;41(3):371-5.

29. Rullo R, Addabbo F, Papaccio G, D'Aquino R, Festa VM. Piezoelectric device vs. conventional rotative instruments in impacted third molar surgery: relationships between surgical difficulty and postoperative pain with histological evaluations. J Craniomaxillofac Surg. 2013 Mar;41(2):e33-8.

30. Piersanti L, Dilorenzo M, Monaco G, Marchetti C. Piezosurgery or conventional rotatory instruments for inferior third molar extractions? J Oral Maxillofac Surg. 2014 Sep;72(9):1647-52.

31. Stübinger S, von Rechenberg vB, Zeilhofer HF, Sader R, Landes C. Er:YAG laser osteotomy for removal of impacted teeth: clinical comparison of two techniques. Lasers Surg Med. 2007 Aug;39(7):583-8.

32. Krausz A, Machtei E, Peled M. Effects of lower third molar extraction on attachment level and alveolar bone height on the adjacent second molar. Int J Oral Maxillofac Surg. 2005;34:756.

33. Amin M, Haria S, Bounds G. Surgical access to an impacted lower third molar by sagittal splitting of the mandible: a case report. Dent Update. 1995;22:206-8.

34. Tay Andrew BG. Buccal corticotomy for removal of deeply impacted mandibular molars. Br J Oral Maxillofac Surg. 2007;45:83-4.

35. Ward TG. The split bone technique for removal of lower third molars. Br Dent J. 1956;101:297.

36. Farish SE, Bouloux GF. General technique of third molar removal. Oral Maxillofacial Surg Clin N Am. 2007;19:23-43.

37. Henry CB. Excision of the developing mandibular third molar by lateral trepanation. Br Dent J. 1969;127:111-8.

38. Chiapasco M, Crescentini M, Romanoni G. Germectomy or delayed removal of mandibular impacted third molars: the relationship between age and incidence of complications. J Oral Maxillofac Surg. 1995;53(4):418-22.

39. Pogrel MA. Partial odontectomy. Oral Maxillofacial Surg Clin N Am. 2007;19:85-91.

40. Checchi I, Alessandri Bonetti G, Pelliccioni GA. Removing high risk impacted mandibular third molars: a surgical-orthodontic approach. J Am Dent Assoc. 1996;127:1214.

41. Alessandri Bonetti G, Bendandi M, Checchi V, Checchi I. Orthodontic extraction: riskless extraction of impacted lower third molars close to the mandibular canal. J Oral Maxillofac Surg. 2007;65(12):2580-6.

42. Peterson LJ, Ellis E, Hupp JR, Tucker MR. Contemporary oral and maxillofacial surgery. 4th ed. St. Louis: Mosby; 2003. p. 185-237.

43. Bouloux Gary F, Steed Martin B, Perciaccante VJ. Complications of third molar surgery. Oral Maxillofacial Surg Clin N Am. 2007;19:117-28.

44. Pogrel M. Complications of third molar surgery. Oral Maxillofac Surg Clin North Am. 1990;2:441. 
45. Matthew I. What should I do if a maxillary third molar is inadvertently displaced into the adjacent soft tissues during surgical removal? [point of care]. J Can Dent Assoc. 2007;73(4):311-2.

46. Dimitrakopoulos I, Papadaki M. Displacement of a maxillary third molar into the infratemporal fossa: case report. Quintessence Int. 2007;38(7):607-10.

47. Haug RH, Perrott DH, Gonzalez ML, Talwar RM. The American association of oral and maxillofacial surgeons age-related third molar study. J Oral Maxillofac Surg. 2005;63(8):1106-14.

48. Pasqualini D, Cocero N, Castella A, Mela L, Bracco P. Primary and secondary closure of the surgical wound after removal of impacted mandibular third molars: a comparative study. Int J Oral Maxillofac Surg. 2005 Jan;34(1):52-7.

49. Rakprasitkul S, Pairuchvej V. Mandibular third molar surgery with primary closure and tube drain. Int J Oral Maxillofac Surg. 1997 Jun;26(3):187-90.

50. Simon D, Manuel S, Geetha V, Naik BR. Potential for osseous regeneration of platelet-rich plasma--a comparative study in mandibular third molar sockets. Indian J Dent Res. 2004 Oct-Dec;15(4):133-6.

51. Varghese MP, Manuel S, Kumar LKS. Potential for osseous regeneration of platelet-rich fibrin-a comparative study in mandibu- lar third molar impaction sockets. J Oral Maxillofac Surg. 2017 Jul;75(7):1322-9.

52. Canellas JVDS, Medeiros PJD, Figueredo CMDS, Fischer RG, Ritto FG. Platelet-rich fibrin in oral surgical procedures: a systematic review and meta-analysis. Int J Oral Maxillofac Surg. 2019 Mar;48(3):395-414.

53. Al-Hamed FS, Tawfik MA, Abdelfadil E, Al-Saleh MAQ. Efficacy of platelet-rich fibrin after mandibular third molar extraction: a systematic review and meta-analysis. J Oral Maxillofac Surg. 2017 Jun;75(6):1124-35.

54. Dodson T. Is there a role for reconstructive techniques to prevent periodontal defects after third molar surgery. J Oral Maxillofac Surg. 2005;63:891.

55. Task Force for Third Molar Summary. Summary of the third molar clinical trials: report of the AAOMS task force for third molar summary. J Oral Maxillofac Surg. 2012 Sep;70(9):2238-48.

56. https://www.nice.org.uk/guidance/ta1

57. https://www.aaoms.org/docs/govt_affairs/advocacy_white_papers/ white_paper_third_molar_data.pdf

Open Access This chapter is licensed under the terms of the Creative Commons Attribution 4.0 International License (http://creativecommons. org/licenses/by/4.0/), which permits use, sharing, adaptation, distribution and reproduction in any medium or format, as long as you give appropriate credit to the original author(s) and the source, provide a link to the Creative Commons license and indicate if changes were made.

The images or other third party material in this chapter are included in the chapter's Creative Commons license, unless indicated otherwise in a credit line to the material. If material is not included in the chapter's Creative Commons license and your intended use is not permitted by statutory regulation or exceeds the permitted use, you will need to obtain permission directly from the copyright holder. 